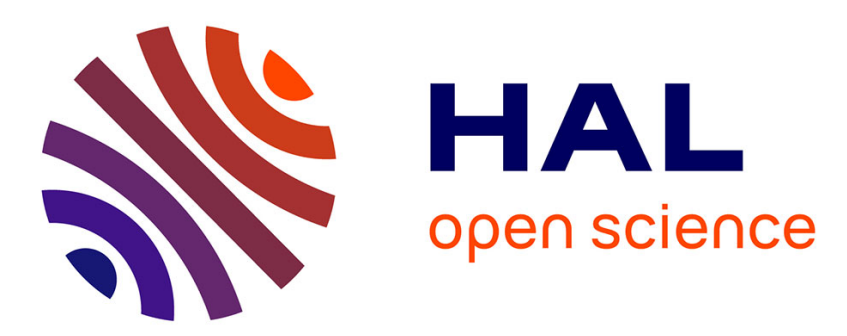

\title{
Generalized probabilistic approach of uncertainties in computational dynamics using random matrices and polynomial chaos decompositions
}

Christian Soize

\section{- To cite this version:}

Christian Soize. Generalized probabilistic approach of uncertainties in computational dynamics using random matrices and polynomial chaos decompositions. International Journal for Numerical Methods in Engineering, 2010, 81 (8), pp.939-970. 10.1002/nme.2712 . hal-00684322

\section{HAL Id: hal-00684322 \\ https://hal.science/hal-00684322}

Submitted on 1 Apr 2012

HAL is a multi-disciplinary open access archive for the deposit and dissemination of scientific research documents, whether they are published or not. The documents may come from teaching and research institutions in France or abroad, or from public or private research centers.
L'archive ouverte pluridisciplinaire HAL, est destinée au dépôt et à la diffusion de documents scientifiques de niveau recherche, publiés ou non, émanant des établissements d'enseignement et de recherche français ou étrangers, des laboratoires publics ou privés. 


\title{
Generalized probabilistic approach of uncertainties in computational dynamics using random matrices and polynomial chaos decompositions
}

\author{
Christian Soize ${ }^{1}$ \\ ${ }^{1}$ Université Paris-Est, Laboratoire de Modelisation et Simulation Multi-Echelle, MSME FRE3160 CNRS, \\ 5 bd Descartes, 77454 Marne-la-Vallee, France
}

\section{SUMMARY}

A new generalized probabilistic approach of uncertainties is proposed for computational model in structural linear dynamics and can be extended without difficulty to computational linear vibroacoustics and to computational nonlinear structural dynamics. This method allows the prior probability model of each type of uncertainties (model-parameter uncertainties and modeling errors) to be separately constructed and identified. The modeling errors are not taken into account with the usual output-prediction-error method but with the nonparametric probabilistic approach of modeling errors recently introduced and based on the use of the random matrix theory. The theory, an identification procedure and a numerical validation are presented. Then a chaos decomposition with random coefficients is proposed to represent the prior probabilistic model of random responses. The random germ is related to the prior probability model of model-parameter uncertainties. The random coefficients are related to the prior probability model of modeling errors and then depends on the random matrices introduced by the nonparametric probabilistic approach of modeling errors. A validation is presented. Finally, a future perspective is introduced when experimental data are available. The prior probability model of the random coefficients can be improved in constructing a posterior probability model using the Bayesian approach.

Copyright @ 2009 John Wiley \& Sons, Ltd.

KEY WORDS: Uncertainties; Computational dynamics; Random matrix; Polynomial chaos

\section{Introduction}

This paper is devoted to the presentation of a generalized probabilistic approach which allows an independent modeling of both model-parameter uncertainties and modeling errors to be performed for computational dynamical models in structural dynamics, structural acoustics, vibration or vibroacoustics, for linear or nonlinear problems. This means that we consider a computational dynamical model of a real system as a selected class of mathematical models

${ }^{*}$ Correspondence to: Christian Soize, Université Paris-Est, Laboratoire de Modelisation et Simulation MultiEechelle, MSME FRE3160 CNRS, 5 bd Descartes, 77454 Marne-la-Vallee, France

Copyright (c) 2009 John Wiley \& Sons, Ltd. 
with an input and an output and depending on a model parameter and on a design parameter. Once the class of computational models has been selected, two types of uncertainties can be identified. The first type is related to the model-parameter uncertainties which mean that the model parameter is not exactly known and is uncertain. The second type is due to modeling errors which are introduced by the mechanical-mathematical process allowing the computational dynamical model to be constructed. The modeling errors introduce model uncertainties in the response predictions constructed with any model belonging to the selected class. This second type of uncertainties will be called model uncertainties. It should be noted that the robust design optimization consists in finding the optimal value of the design parameter which maximizes a cost function related to the model output predicted with the computational dynamical model for which uncertainties are modeled.

Various methods exist for assessing uncertainties in a model. These methods are either probabilistic or deterministic (see for instance [25], [37]). Today, it is well understood that the probabilistic approach of uncertainties must be used as soon as the probability theory can be applied. In this paper, we limit the developments to the cases for which the probability theory can be used to model uncertainties. The construction of the probabilistic model of uncertainties is a fundamental problem which must carefully carried out in order to improve the quality of the predictions of the computational model but also to solve in the best conditions robust design optimization (see for instance [24], [6], [38]).

A model chosen in the selected class will be called the mean model. For given nominal values of the model parameter, the mean model is often called the nominal model. The uncertainties are then related to the mean model. For the construction of the probabilistic model of uncertainties, two main cases have to be considered. For the first one, no experiment of the real system is assumed to be available in order to update the model and to identify the prior probabilistic model of uncertainties. In this case, the prior probabilistic model must have the capability to take into account model uncertainties because there are no possibilities to improve the prior probabilistic model using experiments. In opposite, for the second one, some experiments are assumed to be available to update the mean model [49], [53], to identify the prior probabilistic model of uncertainties [52], [53], [9], [50] and to construct a posterior probabilistic model (see for instance [4] [52], [22]). Today, the first case is a frequent situation encountered for the design and the optimization of complex mechanical systems and has to be carefully analyzed. This means that no experiment is available and that there is neither possibility to update the mean model with experiments nor to identify the prior probability model of uncertainties using mathematical statistics such as the maximum likelihood method or the Bayesian method. In these two cases, the prior probabilistic approach of uncertainties which has to be developed must have the capability to represent modeling errors for the family of models spanned when the design parameter runs through all its admissible set.

Concerning model-parameter uncertainties, the main method is based on the used of the parametric probabilistic approach which has extensively been developed in the last three decades, which is still in development and which allows the uncertain model parameters of the mean model to be taken into account through the introduction of a prior probability model of these model parameters (see for instance [16], [2], [27], [36], [47], [3], [32]). Such an approach consists in modeling uncertain model parameters by a vector-valued random variable (this random vector can correspond to the finite approximation of a random field). The prior stochastic modeling of model-parameter uncertainties then consists (1) either in constructing an adapted representation based on a polynomial chaos decomposition (see for instance [16], 
[19], [17], [26], [44], [18], [36], [10], [9], [20]) (2) or in directly constructing the probability distribution of the random quantity using the available information and the Maximum Entropy Principle introduced by Jaynes [21] in the context of Information Theory developed by Shannon [40] (see for instance [23], [22] and for recent developments in this field [48]).

Concerning model uncertainties induced by modeling errors, it is today well understood that prior and posterior probabilistic models of the uncertain model parameter are not sufficient and do not have the capability to take into account model uncertainties as explained in the context of computational mechanics (see for instance [4], [41], [45], [46]). Two main methods can be used to take into account model uncertainties (modeling errors).

(i) The first one consists in introducing a probabilistic model of the output prediction error which is the difference between the real system output and the model output (note that such a probabilistic approach of model uncertainties is implemented at the output level of the mean model and not implemented at the operator level of the model). When experiments are available, the observed prediction error is then the difference between the measured real system output and the model output. A posterior probabilistic model can be constructed using, for instance, the Bayesian approach (see for instance [4]). With such a method, it is usually assumed that the measurement noise is negligible compared with the prediction error. The advantage of such an approach is its simplicity and its efficiency when simultaneously, a lot of experiments are available and the design parameters are fixed. However, such an approach is not really adapted if the design parameters are not fixed but have to run through an admissible set of values in the context of robust design optimization. Another reason is necessity that modeling errors be taken into account at the operator level of the mean model, for instance to take into account the mass and stiffness operators in order to analyze the generalized eigenvalue problem related to a dynamical system. In this case, the output-prediction-error method is not really adapted to take into account modeling errors.

(ii) The second one is based on the nonparametric probabilistic approach of model uncertainties (modeling errors) which has recently been proposed in [41] as another possible way to the use of the output-prediction-error method in order to take into account modeling errors. The nonparametric probabilistic approach consists in directly constructing the stochastic modeling of the operators of the mean computational model instead of introducing a probabilistic model of the prediction errors. The random matrix theory (see for instance [1] and [29]) is used to construct the prior probability distribution of the random matrices modeling the uncertain operators of the mean model are constructed using again the Maximum Entropy Principle for which the constraints are defined by the available information [41], [42], [43], [45], [46]. Since the paper [41], many works have been published in order to validate the nonparametric probabilistic approach of model uncertainties with experimental results (see for instance [7], [46], [8], [12], [13], [50], [14]) and to extend the theory, in particular, with the introduction of a new set of positive-definite random matrices yielding a more flexible description of the dispersion levels [30], with the analysis of complex vibroacoustic systems in the mediumfrequency range [13], [14], with the analysis of nonlinear dynamical systems for local nonlinear elements [11], [35] and for distributed nonlinear elements or nonlinear geometrical effects [31].

It should be noted that the major difference between the two approaches (i) and (ii) described above is due to the fact that the statistical fluctuations of responses generated by the output-prediction-error method are independent of the state variable of the dynamical system while the statistical fluctuations of responses generated by the nonparametric probabilistic 
approach depend on it. Approach (ii) has been proposed to avoid the difficulty induced in (i). As it has been proven in [41], [45] and [46], the nonparametric probabilistic approach has the capability to simultaneously take into account both model-parameter uncertainties and modeling errors. With such an approach, for each random matrix of the stochastic reduced computational model such as the generalized mass matrix for instance, the level of uncertainties induced by both model-parameter uncertainties and modeling errors are controlled by only one dispersion parameter. Consequently, with such an approach, the level of uncertainties for the model parameter cannot be separated from the level of uncertainties induced by modeling errors. In addition, in the nonparametric probabilistic approach and by construction, the mean value of each random matrix is chosen as the matrix of the reduced mean computational model associated with the nominal value. Clearly, this point could be improved in choosing the mean value of each random matrix as the mean matrix of the stochastic computational model induced only by the parametric probabilistic approach of model-parameter uncertainties. Such a method should required again to separate the probabilistic model of model-parameter uncertainties and of modeling errors. This is the reason why, we propose a generalized probabilistic approach of uncertainties allowing both the modelparameter uncertainties and modeling errors to be simultaneously taken into account but in a separate way. This new method allows the prior probability model of each type of uncertainties (model-parameter uncertainties and modeling errors) to be separately constructed and to be separately identified with experiments if experiments are available. In addition, this method will allow the biais of the mean values of the stochastic responses to be decreased. In a first part of the paper, we present the theory, an identification procedure of the dispersion parameters controlling the level of each type of uncertainties when experimental data are available, and a numerical validation. In a second part, we show how the chaos decomposition with random coefficients [51] can be used to represent the prior probabilistic model of random responses of the stochastic computational model. The random germ of the chaos decomposition is related to the prior probabilistic model of model-parameter uncertainties. The random coefficients of the decomposition are related to the prior probability model of modeling errors and then depends on the random matrices introduced by the nonparametric probabilistic approach of modeling errors. It can then be deduced that the chaos are then statistically independent of the random coefficients of the chaos decomposition. Finally, when experimental data are available, a posterior probability model of these random coefficients is proposed using the Bayesian approach.

In order to simply explain all the aspects of this new method, we have chosen to present the developments for the most simple context of structural linear dynamics. The extension to other cases is straightforward in particular:

- for computational vibroacoustics in low- and medium-frequency ranges with modeling errors in the structure, in the acoustic cavity, for the vibroacoustic coupling operator and for insulation schemes (see [13], [14])

- for computational nonlinear structural dynamics with local or distributed nonlinear elements [11], [35] or with nonlinear geometrical effects in the general context of threedimensional nonlinear electrodynamics [31]. 
2. Formulation of a prior generalized probabilistic approach of uncertainties

\subsection{Introduction of the mean computational model}

As explained at the end of Section 1, in order to simply explain the method proposed, we have chosen to limit the developments to computational models of linear dynamical systems made up of a three-dimensional damped fixed structure around a static equilibrium configuration considered as a natural state without prestresses and subjected to an external load. For given nominal values of the parameters of the dynamical system, the basic finite element model is called the mean computational model. In addition, it is assumed that a set of model parameters has been identified as uncertain model parameters. These model parameters are the components of a vector $\mathbf{x}=\left(x_{1}, \ldots, x_{n_{p}}\right)$ belonging to an admissible set $\mathcal{C}_{\text {par }}$ which is a subset of $\mathbb{R}^{n_{p}}$. The linear dynamical equation of the mean computational model is then written as

$$
[\mathbb{M}(\mathbf{x})] \ddot{\mathbf{y}}(t)+[\mathbb{D}(\mathbf{x})] \dot{\mathbf{y}}(t)+[\mathbb{K}(\mathbf{x})] \mathbf{y}(t)=\mathbf{f}(t ; \mathbf{x}),
$$

in which $\mathbf{y}=\left(y_{1}, \ldots, y_{m}\right)$ is the unknown time response vector of the $m$ degrees of freedom (DOF) (displacements and/or rotations); $\dot{\mathbf{y}}$ and $\ddot{\mathbf{y}}$ are the velocity and acceleration vectors respectively; $\mathbf{f}(t ; \mathbf{x})=\left(f_{1}(t ; \mathbf{x}), \ldots, f_{m}(t ; \mathbf{x})\right)$ is the known external load vector of the $m$ inputs (forces and/or moments); $[\mathbb{M}(\mathbf{x})],[\mathbb{D}(\mathbf{x})]$ and $[\mathbb{K}(\mathbf{x})]$ are the mass, damping and stiffness matrices of the mean computational model, which belong to the set $\mathbb{M}_{m}^{+}(\mathbb{R})$ of all the positivedefinite symmetric $(m \times m)$ real matrices.

It should be noted that the corresponding nonlinear dynamical equation of the mean computational model would have been written as

$$
[\mathbb{M}(\mathbf{x})] \ddot{\mathbf{y}}(t)+[\mathbb{D}(\mathbf{x})] \dot{\mathbf{y}}(t)+[\mathbb{K}(\mathbf{x})] \mathbf{y}(t)+\mathbf{f}_{\mathrm{NL}}(\mathbf{y}(t), \dot{\mathbf{y}}(t) ; \mathbf{x})=\mathbf{f}(t ; \mathbf{x}),
$$

in which $(\mathbf{y}, \mathbf{z}) \mapsto \mathbf{f}_{\mathrm{NL}}(\mathbf{y}, \mathbf{z})$ would have been a nonlinear mapping from $\mathbb{R}^{m} \times \mathbb{R}^{m}$ into $\mathbb{R}^{m}$ modeling additional nonlinear damping and restoring forces. As we have explained above, we consider now the linear case taking $\mathbf{f}_{\mathrm{NL}}=0$ and yielding Eq. (1).

\subsection{Introduction of the reduced mean computational model}

There are two main possibilities to construct the reduced mean computational model in the context of the parametric probabilistic approach of model-parameter uncertainties for which parameter $\mathbf{x}$ is modeled by a random variable $\mathbf{X}$. The mean value of $\mathbf{X}$ will be the nominal value $\underline{\mathbf{x}}=\left(\underline{x}_{1}, \ldots, \underline{x}_{n_{p}}\right)$ of the uncertain model parameter $\mathbf{x}$ and the support of its probability distribution on $\mathbb{R}^{n_{p}}$ is $\mathcal{C}_{\text {par }}$.

(1) The first one consists in solving the generalized eigenvalue problem associated with the mean mass and stiffness matrices for $\mathbf{x}$ fixed to its nominal value $\underline{\mathbf{x}}$. We then obtain the elastic modes of the nominal mean computational model which are independent of $\mathbf{x}$ and which depend only on $\underline{\mathbf{x}}$ which is fixed. In this case, when $\mathbf{x}$ runs through $\mathcal{C}_{\text {par }}$, matrices $[M(\mathbf{x})]$ and $[\mathbb{K}(\mathbf{x})]$ have to be projected on the subspace spanned by the elastic modes of the nominal mean computational model. For very large computational model ( $m$ can be several tens of millions) such an operation is not easy to perform with usual commercial softwares which often are black boxes.

(2) The second one consists in solving the generalized eigenvalue problem associated with the mean mass and stiffness matrices for each required $\mathbf{x}$ belonging to $\mathcal{C}_{\text {par }}$. In this case, the 
elastic modes of the mean computational model depend on $\mathbf{x}$. In the context of the parametric probabilistic approach of model-parameter uncertainties, we then have to solve a random generalized eigenvalue problem and such an approach is better adapted to usual commercial softwares and allows a fast convergence to be obtained with respect to the reduced order dimension. In addition, some algorithms have been developed in this context for random eigenvalue problems of large systems [33]. In order to limit the developments, we will focus the presentation using this second approach. The extension to the first approach is straightforward from a theoretical point of view (see for instance [41]). Finally, it should be noted that the random generalized eigenvalue problem can also be considered in a polynomial chaos decomposition for which an efficient approach has been proposed [19]. Such an ingredient can be added without difficulty in the developments presented below but would induce an addition degree of difficulty in the understanding which could mask the ideas of the method proposed.

For each value of $\mathbf{x}$ given in $\mathcal{C}_{\text {par }}$, the generalized eigenvalue problem associated with the mean mass and stiffness matrices is written as

$$
[\mathbb{K}(\mathbf{x})] \varphi(\mathbf{x})=\lambda(\mathbf{x})[\mathbb{M}(\mathbf{x})] \varphi(\mathbf{x}),
$$

for which the eigenvalues $0<\lambda_{1}(\mathbf{x}) \leq \lambda_{2}(\mathbf{x}) \leq \ldots \leq \lambda_{m}(\mathbf{x})$ and the associated elastic modes $\left\{\varphi_{1}(\mathbf{x}), \varphi_{2}(\mathbf{x}), \ldots\right\}$ are such that

$$
<[\mathbb{M}(\mathbf{x})] \varphi_{\alpha}(\mathbf{x}), \varphi_{\beta}(\mathbf{x})>=\mu_{\alpha}(\mathbf{x}) \delta_{\alpha \beta} \quad, \quad<[\mathbb{K}(\mathbf{x})] \varphi_{\alpha}(\mathbf{x}), \varphi_{\beta}(\mathbf{x})>=\mu_{\alpha}(\mathbf{x}) \omega_{\alpha}(\mathbf{x})^{2} \delta_{\alpha \beta},
$$

in which $\omega_{\alpha}(\mathbf{x})=\sqrt{\lambda_{\alpha}(\mathbf{x})}$ is the eigenfrequency of elastic mode $\varphi_{\alpha}(\mathbf{x})$ whose normalization is defined by the generalized mass $\mu_{\alpha}(\mathbf{x})$ and where $\langle\mathbf{u}, \mathbf{v}\rangle=\sum_{j} u_{j} v_{j}$ is the Euclidean inner product of the vectors $\mathbf{u}$ and $\mathbf{v}$.

For each value of $\mathbf{x}$ given in $\mathcal{C}_{\mathrm{par}}$, the reduced mean computational model of the dynamical system is obtained in constructing the projection of the mean computational model on the subspace $\mathbb{H}_{n}$ of $\mathbb{R}^{m}$ spanned by $\left\{\varphi_{1}(\mathbf{x}), \ldots, \varphi_{n}(\mathbf{x})\right\}$ with $n \ll m$. Let $\left[\phi_{n}(\mathbf{x})\right]$ be the $(m \times n)$ real matrix whose columns are vectors $\left\{\varphi_{1}(\mathbf{x}), \ldots, \varphi_{n}(\mathbf{x})\right\}$. The generalized force $\mathbf{f}^{n}(t ; \mathbf{x})$ is an $\mathbb{R}^{n}$-vector such that $\mathbf{f}^{n}(t ; \mathbf{x})=\left[\phi_{n}(\mathbf{x})\right]^{T} \mathbf{f}(t ; \mathbf{x})$. For all $\mathbf{x}$ in $\mathcal{C}_{\text {par }}$, the generalized mass, damping and stiffness matrices $\left[M_{n}(\mathbf{x})\right],\left[D_{n}(\mathbf{x})\right]$ and $\left[K_{n}(\mathbf{x})\right]$ belong to the set $\mathbb{M}_{n}^{+}(\mathbb{R})$ and are defined by

$$
\left[M_{n}(\mathbf{x})\right]_{\alpha \beta}=\mu_{\alpha}(\mathbf{x}) \delta_{\alpha \beta},\left[D_{n}(\mathbf{x})\right]_{\alpha \beta}=<[\mathbb{D}(\mathbf{x})] \varphi_{\beta}(\mathbf{x}), \varphi_{\alpha}(\mathbf{x})>,\left[K_{n}(\mathbf{x})\right]_{\alpha \beta}=\mu_{\alpha}(\mathbf{x}) \omega_{\alpha}(\mathbf{x})^{2} \delta_{\alpha \beta},
$$

in which, generally, $\left[D_{n}(\mathbf{x})\right]$ is a full matrix. Consequently, for all $\mathbf{x}$ in $\mathcal{C}_{\text {par }}$ and for all fixed $t$, the reduced mean computational model of the dynamical system is written as the projection $\mathbf{y}^{n}(t)$ of $\mathbf{y}(t)$ on $\mathbb{H}_{n}$ which can be written as $\mathbf{y}^{n}(t)=\left[\phi_{n}(\mathbf{x})\right] \mathbf{q}^{n}(t)$ in which the vector $\mathbf{q}^{n}(t) \in \mathbb{R}^{n}$ of the generalized coordinates verifies the differential equation,

$$
\left[M_{n}(\mathbf{x})\right] \ddot{\mathbf{q}}^{n}(t)+\left[D_{n}(\mathbf{x})\right] \dot{\mathbf{q}}^{n}(t)+\left[K_{n}(\mathbf{x})\right] \mathbf{q}^{n}(t)=\mathbf{f}^{n}(t ; \mathbf{x})
$$

\subsection{Development of a prior generalized probabilistic approach of uncertainties}

The prior generalized probabilistic approach of uncertainties which is proposed consists in simultaneously used the parametric probabilistic approach of model-parameter uncertainties and the nonparametric probabilistic approach of model uncertainties (modeling errors). In this section, the complete methodology is presented. 
2.3.1. Methodology for the construction of the prior generalized probabilistic approach of uncertainties. Let $(\Theta, \mathcal{T}, \mathcal{P})$ and $\left(\Theta^{\prime}, \mathcal{T}^{\prime}, \mathcal{P}^{\prime}\right)$ be two probability spaces. The first one will be devoted to the probabilistic model of model-parameter uncertainties using the parametric probabilistic approach and the second one to the probabilistic model of model uncertainties (modeling errors) using the nonparametric probabilistic approach. Let $\mathbf{X}=\{\theta \mapsto \mathbf{X}(\theta)\}$ be a random variable defined on $(\Theta, \mathcal{T}, \mathcal{P})$ and let $[\mathbf{G}]=\left\{\theta^{\prime} \mapsto\left[\mathbf{G}\left(\theta^{\prime}\right)\right]\right\}$ be another random variable defined on $\left(\Theta^{\prime}, \mathcal{T}^{\prime}, \mathcal{P}^{\prime}\right)$. The two random variables $\mathbf{X}$ and $[\mathbf{G}]$ are then independent and their mathematical expectations are such that

$$
E\{\mathbf{X}\}=\int_{\Theta} \mathbf{X}(\theta) d \mathcal{P}(\theta) \quad, \quad E\{[\mathbf{G}]\}=\int_{\Theta^{\prime}}\left[\mathbf{G}\left(\theta^{\prime}\right)\right] d \mathcal{P}^{\prime}\left(\theta^{\prime}\right)
$$

If $\mathbf{Q}=\mathbf{h}(\mathbf{X},[\mathbf{G}])$ is a random variable defined by a given deterministic transformation $\mathbf{h}$ of independent random variables $\mathbf{X}$ and $[\mathbf{G}]$, then the mathematical expectation of $\mathbf{Q}$ is

$$
E\{\mathbf{Q}\}=\int_{\Theta} \int_{\Theta^{\prime}} \mathbf{h}\left(\mathbf{X}(\theta),\left[\mathbf{G}\left(\theta^{\prime}\right)\right]\right) d \mathcal{P}^{\prime}\left(\theta^{\prime}\right) d \mathcal{P}(\theta)
$$

(1) The first step of the generalized probabilistic approach of uncertainties consists in constructing the probabilistic model of model-parameter uncertainties for which parameter $\mathbf{x}$ is modeled by a random variable $\mathbf{X}$ defined on probability space $(\Theta, \mathcal{T}, \mathcal{P})$. Consequently, the generalized matrices in Eq. (5) become random matrices $\left[M_{n}(\mathbf{X})\right],\left[D_{n}(\mathbf{X})\right]$ and $\left[K_{n}(\mathbf{X})\right]$ and, for all fixed $t$, the generalized external force $\mathbf{f}^{n}(t ; \mathbf{x})$ becomes a random vector $\mathbf{f}^{n}(t ; \mathbf{X})$. The mean values of these random matrices are denoted by $\left[\underline{M}_{n}\right],\left[\underline{D}_{n}\right]$ and $\left[\underline{K}_{n}\right]$. We then have

$$
E\left\{\left[M_{n}(\mathbf{X})\right]\right\}=\left[\underline{M}_{n}\right] \quad, \quad E\left\{\left[D_{n}(\mathbf{X})\right]\right\}=\left[\underline{D}_{n}\right] \quad, \quad E\left\{\left[K_{n}(\mathbf{X})\right]\right\}=\left[\underline{K}_{n}\right] \quad .
$$

It should be noted that the mean matrices $\left[\underline{M}_{n}\right],\left[\underline{D}_{n}\right]$ and $\left[\underline{K}_{n}\right]$ are different from the matrices $\left[M_{n}(\underline{\mathbf{x}})\right],\left[D_{n}(\underline{\mathbf{x}})\right]$ and $\left[K_{n}(\underline{\mathbf{x}})\right]$ of the nominal mean computational model.

(2) The second step of the generalized probabilistic approach of uncertainties consists in constructing the probabilistic model of model uncertainties (modeling errors) in using the nonparametric probabilistic approach [41] (see the additional explanations given in Section 2.3.4). Therefore, for all $\mathbf{x}$ fixed in $\mathcal{C}_{\text {par }}$, the matrices $\left[M_{n}(\mathbf{x})\right],\left[D_{n}(\mathbf{x})\right]$ and $\left[K_{n}(\mathbf{x})\right]$ are replaced by independent random matrices

$$
\left[\mathbf{M}_{n}(\mathbf{x})\right]=\left\{\theta^{\prime} \mapsto\left[\mathbf{M}_{n}\left(\theta^{\prime} ; \mathbf{x}\right)\right]\right\},\left[\mathbf{D}_{n}(\mathbf{x})\right]=\left\{\theta^{\prime} \mapsto\left[\mathbf{D}_{n}\left(\theta^{\prime} ; \mathbf{x}\right)\right]\right\},\left[\mathbf{K}_{n}(\mathbf{x})\right]=\left\{\theta^{\prime} \mapsto\left[\mathbf{K}_{n}\left(\theta^{\prime} ; \mathbf{x}\right)\right]\right\}
$$

defined on probability space $\left(\Theta^{\prime}, \mathcal{T}^{\prime}, \mathcal{P}^{\prime}\right)$ and belonging to the set of random matrices introduced in [41] and [46], or in [30]. In order to simplify the presentation, we will limit the developments to the case for which these random matrices belong to the set $\mathrm{SE}^{+}$of random matrices introduced in [46]. The extension to the use of the set introduced in [30] is straightforward. The mathematical expectation of these random matrices must be such that (see the reasons developed in Section 2.3.4),

$$
E\left\{\left[\mathbf{M}_{n}(\mathbf{x})\right]\right\}=\left[M_{n}(\mathbf{x})\right] \quad, \quad E\left\{\left[\mathbf{D}_{n}(\mathbf{x})\right]\right\}=\left[D_{n}(\mathbf{x})\right] \quad, \quad E\left\{\left[\mathbf{K}_{n}(\mathbf{x})\right]\right\}=\left[K_{n}(\mathbf{x})\right] .
$$

(3) The last step of the construction of the generalized probabilistic approach of uncertainties 
then consists in replacing in Eq. (5) $\mathbf{x}$ by $\mathbf{X}$ and in replacing the dependent random matrices $\left[M_{n}(\mathbf{X})\right],\left[D_{n}(\mathbf{X})\right]$ and $\left[K_{n}(\mathbf{X})\right]$ by the dependent random matrices

$$
\begin{aligned}
{\left[\mathbf{M}_{n}(\mathbf{X})\right] } & =\left\{\left(\theta, \theta^{\prime}\right) \mapsto\left[\mathbf{M}_{n}\left(\theta^{\prime} ; \mathbf{X}(\theta)\right)\right]\right\} \\
{\left[\mathbf{D}_{n}(\mathbf{X})\right] } & =\left\{\left(\theta, \theta^{\prime}\right) \mapsto\left[\mathbf{D}_{n}\left(\theta^{\prime} ; \mathbf{X}(\theta)\right)\right]\right\} \\
{\left[\mathbf{K}_{n}(\mathbf{X})\right] } & =\left\{\left(\theta, \theta^{\prime}\right) \mapsto\left[\mathbf{K}_{n}\left(\theta^{\prime} ; \mathbf{X}(\theta)\right)\right]\right\}
\end{aligned}
$$

defined on the probability space $\left(\Theta \times \Theta^{\prime}, \mathcal{T} \otimes \mathcal{T}^{\prime}, \mathcal{P} \otimes \mathcal{P}^{\prime}\right)$. From Eqs. (8) and (10), it can easily be deduced that

$$
E\left\{\left[\mathbf{M}_{n}(\mathbf{X})\right]\right\}=\left[\underline{M}_{n}\right] \quad, \quad E\left\{\left[\mathbf{D}_{n}(\mathbf{X})\right]\right\}=\left[\underline{D}_{n}\right] \quad, \quad E\left\{\left[\mathbf{K}_{n}(\mathbf{X})\right]\right\}=\left[\underline{K}_{n}\right] \quad .
$$

2.3.2. Stochastic reduced computation model generated by the prior generalized probabilistic approach of uncertainties. The generalized probabilistic approach of uncertainties consists in replacing the mean computational model by the following stochastic reduced computational model,

$$
\begin{gathered}
\mathbf{Y}^{n}(t)=\left[\phi_{n}(\mathbf{X})\right] \mathbf{Q}^{n}(t) \\
{\left[\mathbf{M}_{n}(\mathbf{X})\right] \ddot{\mathbf{Q}}^{n}(t)+\left[\mathbf{D}_{n}(\mathbf{X})\right] \dot{\mathbf{Q}}^{n}(t)+\left[\mathbf{K}_{n}(\mathbf{X})\right] \mathbf{Q}^{n}(t)=\mathbf{f}^{n}(t ; \mathbf{X})}
\end{gathered}
$$

in which for all fixed $t, \mathbf{Y}^{n}(t)=\left\{\left(\theta, \theta^{\prime}\right) \mapsto \mathbf{Y}^{n}\left(\theta, \theta^{\prime} ; t\right)\right\}$ is an $\mathbb{R}^{m}$-valued random vector and $\mathbf{Q}^{n}(t)=\left\{\left(\theta, \theta^{\prime}\right) \mapsto \mathbf{Q}^{n}\left(\theta, \theta^{\prime} ; t\right)\right\}$ is an $\mathbb{R}^{n}$-valued random vector defined on $\left(\Theta \times \Theta^{\prime}, \mathcal{T} \otimes \mathcal{T}^{\prime}, \mathcal{P} \otimes\right.$ $\left.\mathcal{P}^{\prime}\right)$. Thus, for any realization $\mathbf{X}(\theta)$ of random variable $\mathbf{X}$ with $\theta$ in $\Theta$, and for any realization $\left[\mathbf{M}_{n}\left(\theta^{\prime} ; \mathbf{x}\right)\right],\left[\mathbf{D}_{n}\left(\theta^{\prime} ; \mathbf{x}\right)\right],\left[\mathbf{K}_{n}\left(\theta^{\prime} ; \mathbf{x}\right)\right]$ of independent random matrices $\left[\mathbf{M}_{n}(\mathbf{x})\right],\left[\mathbf{D}_{n}(\mathbf{x})\right],\left[\mathbf{K}_{n}(\mathbf{x})\right]$ for $\theta^{\prime}$ in $\Theta^{\prime}$ and $\mathbf{x}$ in $\mathcal{C}_{\text {par }}$, the realization $\mathbf{Y}^{n}\left(\theta, \theta^{\prime} ; t\right)$ of the random variable $\mathbf{Y}^{n}(t)$ and the realization $\mathbf{Q}^{n}\left(\theta, \theta^{\prime} ; t\right)$ of the random variable $\mathbf{Q}^{n}(t)$ verify the deterministic problem

$$
\mathbf{Y}^{n}\left(\theta, \theta^{\prime} ; t\right)=\left[\phi_{n}(\mathbf{X}(\theta)] \mathbf{Q}^{n}\left(\theta, \theta^{\prime} ; t\right)\right.
$$

$\left[\mathbf{M}_{n}\left(\theta^{\prime} ; \mathbf{X}(\theta)\right)\right] \ddot{\mathbf{Q}}^{n}\left(\theta, \theta^{\prime} ; t\right)+\left[\mathbf{D}_{n}\left(\theta^{\prime} ; \mathbf{X}(\theta)\right)\right] \dot{\mathbf{Q}}^{n}\left(\theta, \theta^{\prime} ; t\right)+\left[\mathbf{K}_{n}\left(\theta^{\prime} ; \mathbf{X}(\theta)\right)\right] \mathbf{Q}^{n}\left(\theta, \theta^{\prime} ; t\right)=\mathbf{f}^{n}(t ; \mathbf{X}(\theta))$.

2.3.3. Construction of the prior probability model of model-parameter uncertainties. The uncertain model parameter $\mathbf{x}$ (whose nominal value is $\underline{\mathbf{x}}$ ) is modeled by a random variable $\mathbf{X}=\left(X_{1}, \ldots, X_{n_{p}}\right)$, defined on probability space $(\Theta, \mathcal{T}, \mathcal{P})$, with values in $\mathbb{R}^{n_{p}}$. The unknown probability distribution of $\mathbf{X}$ which is denoted by $P_{\mathbf{X}}$ is assumed to be defined by a probability density function $\mathbf{x} \mapsto p_{\mathbf{X}}(\mathbf{x})$ from $\mathbb{R}^{n_{p}}$ into $\mathbb{R}^{+}=[0,+\infty$ [ with respect to the Lebesgue measure $d \mathbf{x}=d x_{1} \ldots d x_{n_{p}}$. Under the assumption that no experimental data are available to construct $p_{\mathbf{X}}$, the prior model can be constructed using the maximum entropy principle [21] introduced in the context of Information Theory [40]. For such a construction, the available information has to be defined. Since $\mathbf{x}$ belongs to $\mathcal{C}_{\text {par }}$, the support of $p_{\mathbf{X}}$ must be $\mathcal{C}_{\text {par }}$ and the normalization condition must be verified. We then have,

$$
\operatorname{supp} p_{\mathbf{X}}=\mathcal{C}_{\text {par }} \subset \mathbb{R}^{n_{p}} \quad, \quad \int_{\mathbb{R}^{n_{p}}} p_{\mathbf{X}}(\mathbf{x}) d \mathbf{x}=\int_{\mathcal{C}_{\text {par }}} p_{\mathbf{X}}(\mathbf{x}) d \mathbf{x}=1 .
$$

Since $\underline{\mathbf{x}} \in \mathcal{C}_{\text {par }} \subset \mathbb{R}^{n_{p}}$ of $\mathbf{x}$ is a nominal value, an additional available information consists in writing that the mean value $E\{\mathbf{X}\}$ of $\mathbf{X}$ is equal to $\underline{\mathbf{x}}$ which yields the following constraint equation,

$$
\int_{\mathbb{R}^{n_{p}}} \mathbf{x} p_{\mathbf{X}}(\mathbf{x}) d \mathbf{x}=\underline{\mathbf{x}}
$$


In general, an additional available information can be deduced from the analysis of the mathematical properties of the solution of the stochastic computational model under construction. The random solution $\mathbf{Q}^{n}$ of the stochastic computational model defined by Eq. (14) must be a second-order vector-valued stochastic process (because the dynamical system is stable) which means that, for all $t$, we must have $E\left\{\left\|\mathbf{Q}^{n}(t)\right\|^{2}\right\}<+\infty$. In order that such a property be verified, it is necessary to introduce a constraint which can always be written as the equation $E\{\mathbf{g}(\mathbf{X})\}=\boldsymbol{\gamma}$ on $\mathbb{R}^{\mu}$, in which $\boldsymbol{\gamma}=\left(\gamma_{1}, \ldots, \gamma_{\mu}\right)$ is a given vector in $\mathbb{R}^{\mu}$ with $\mu \geq 1$ and where $\mathbf{x} \mapsto \mathbf{g}(\mathbf{x})=\left(g_{1}(\mathbf{x}), \ldots, g_{\mu}(\mathbf{x})\right)$ is a given measurable mapping from $\mathbb{R}^{n_{p}}$ into $\mathbb{R}^{\mu}$. Consequently, the additional available information defined the following constraint equation,

$$
\int_{\mathbb{R}^{n_{p}}} \mathbf{g}(\mathbf{x}) p_{\mathbf{X}}(\mathbf{x}) d \mathbf{x}=\boldsymbol{\gamma} .
$$

Let $\mathcal{C}$ be the set of all the probability density functions $p_{\mathbf{X}}$ defined on $\mathbb{R}^{n_{p}}$ with values in $\mathbb{R}^{+}$ such that Eqs. (17) to (19) hold. The maximum entropy principle consists in constructing $p_{\mathbf{X}} \in \mathcal{C}$ such that

$$
p_{\mathbf{X}}=\arg \max _{p \in \mathcal{C}} S(p)
$$

in which the entropy $S(p)$ of the probability density function $p$ is defined by

$$
S(p)=-\int_{\mathbb{R}^{n_{p}}} p(\mathbf{x}) \log (p(\mathbf{x})) d \mathbf{x}
$$

where $\log$ is the Neperian logarithm. Such an optimization problem can be solved as explained in [21], [23] if $n_{p}$ is very small (one or a few units). For high dimension case (for instance, $n_{p}$ is several tens or hundreds) advanced methodologies such as the methodology presented in [48] must be used.

Let $\left[A_{n}(\mathbf{X})\right]$ be the random matrix representing any one of the three random matrices $\left[M_{n}(\mathbf{X})\right],\left[D_{n}(\mathbf{X})\right]$ or $\left[K_{n}(\mathbf{X})\right]$ with values in $\mathbb{M}_{n}^{+}(\mathbb{R})$. In order that $E\left\{\left\|\mathbf{Q}^{n}(t)\right\|^{2}\right\}<+\infty$ for all $t$, we will see below that it is necessary that the probabilistic model of $\mathbf{X}$ must be such that, for all second-order $\mathbb{R}^{n}$-valued random variable $\mathbf{Q}$ defined on $(\Theta, \mathcal{T}, \mathcal{P})$, we have,

$$
E\left\{<\left[A_{n}(\mathbf{X})\right] \mathbf{Q}, \mathbf{Q}>\right\} \geq c_{p} E\left\{\|\mathbf{Q}\|^{2}\right\}
$$

in which $c_{p}>0$ is a positive finite real constant and where $\|\mathbf{u}\|=<\mathbf{u}, \mathbf{u}>^{1 / 2}$ is the Euclidean norm of vector $\mathbf{u}$. The constraint defined by Eq. (22) allows an equation of the type defined by Eq. (19) to be constructed in which $\gamma$ is a free parameter. Since Eq. (19) is a vectorial equation of dimension $\mu$, the solution $p_{\mathbf{X}}$ of Eq. (20) depends on the free $\mathbb{R}^{\mu}$-valued parameter $\boldsymbol{\gamma}$. However, parameter $\boldsymbol{\gamma}$ has generally no physical meaning and it is better to express $\boldsymbol{\gamma}$ in terms of an $\mathbb{R}^{\mu}$-valued parameter $\boldsymbol{\delta}_{\mathbf{X}}$ which corresponds to a well defined statistical quantity for random variable $\mathbf{X}$. In general, $\boldsymbol{\delta}_{\mathbf{X}}$ does not run through $\mathbb{R}^{\mu}$ but must belong to an admissible set $\mathcal{C}_{\mathbf{X}}$ which is a subset of $\mathbb{R}^{\mu}$. Consequently, $p_{\mathbf{X}}$ depends on $\mathcal{C}_{\text {par }}, \underline{\mathbf{x}}$ and $\boldsymbol{\delta}_{\mathbf{X}}$. Since $\mathcal{C}_{\text {par }}$ and $\underline{\mathbf{x}}$ are given and since $\boldsymbol{\delta}_{\mathbf{X}}$ is a free parameter, we will indicate the dependence in $\boldsymbol{\delta}_{\mathbf{X}}$ of $p_{\mathbf{X}}$ in writing this probability density function as

$$
\mathbf{x} \mapsto p_{\mathbf{X}}\left(\mathbf{x} ; \boldsymbol{\delta}_{\mathbf{X}}\right) \quad \text { with } \quad \boldsymbol{\delta}_{\mathbf{X}} \in \mathcal{C}_{\mathbf{X}} \subset \mathbb{R}^{\mu}
$$


2.3.4. Construction of the prior probability model of model uncertainties (modeling errors). Let $A$ be the letter $M, D$ or $K$ relatively to the generalized mass, damping or stiffness matrix. In the nonparametric probabilistic approach of both model-parameter uncertainties and model uncertainties (modeling errors) introduced in [41], the probability model of the statistical fluctuations of the random matrix $\left[\mathbf{A}_{n}\right]$ has been constructed around the deterministic nominal value $\left[A_{n}(\underline{\mathbf{x}})\right]$ of the matrix in the nominal computational model. For the generalized approach of uncertainties which is proposed below, since the model-parameter uncertainties are taken into account by the parametric probabilistic approach in introducing the random matrix $\left[A_{n}(\mathbf{X})\right]$, only the model uncertainties (modeling errors) must be taken into account by the nonparametric probabilistic approach of uncertainties. This means that the probability model of the statistical fluctuations of the random matrix $\left[\mathbf{A}_{n}\right]$ due to the model uncertainties must be constructed around the random matrix $\left[A_{n}(\mathbf{X})\right]$ and not around the deterministic nominal value $\left[A_{n}(\underline{\mathbf{x}})\right]$. Such a construction of the probability model of modeling errors is performed below.

Following the methodology of the construction of the nonparametric probabilistic approach (see [41], [42], [46]), for all $\mathbf{x}$ in $\mathcal{C}_{\text {par }}$, the construction of the probability model of random matrices $\left[\mathbf{M}_{n}(\mathbf{x})\right],\left[\mathbf{D}_{n}(\mathbf{x})\right]$ and $\left[\mathbf{K}_{n}(\mathbf{x})\right]$ defined on probability space $\left(\Theta^{\prime}, \mathcal{T}^{\prime}, \mathcal{P}^{\prime}\right)$ is based on the available information deduced from the fundamental properties of the dynamical system and from additional properties required in order that a second-order stochastic solution exists for Eq. (14) (see [42], [46]). These random matrices must be such that, for all $\mathbf{x}$ in $\mathcal{C}_{\text {par }}$,

$$
\left[\mathbf{M}_{n}(\mathbf{x})\right],\left[\mathbf{D}_{n}(\mathbf{x})\right],\left[\mathbf{K}_{n}(\mathbf{x})\right] \in \mathbb{M}_{n}^{+}(\mathbb{R}) \text { a.s. },
$$

the mean values of these random matrices are defined by Eq. (10) and we must have

$$
E\left\{\left\|\left[\mathbf{M}_{n}(\mathbf{x})\right]^{-1}\right\|_{F}^{2}\right\}<+\infty, E\left\{\left\|\left[\mathbf{D}_{n}(\mathbf{x})\right]^{-1}\right\|_{F}^{2}\right\}<+\infty, E\left\{\left\|\left[\mathbf{K}_{n}(\mathbf{x})\right]^{-1}\right\|_{F}^{2}\right\}<+\infty \quad,
$$

in which $\|A\|_{F}$ is the Frobenius norm of $[A]$ such that $\|A\|_{F}^{2}=\operatorname{tr}\left\{[A]^{T}[A]\right\}$ in which $[A]^{T}$ denotes the transpose of $[A]$ and where $\operatorname{tr}\{[B]\}$ is the trace of $[B]$. Since for all $\mathbf{x}$ in $\mathcal{C}_{\text {par }}$, the deterministic matrices $\left[M_{n}(\mathbf{x})\right],\left[D_{n}(\mathbf{x})\right]$ and $\left[K_{n}(\mathbf{x})\right]$ are in $\mathbb{M}_{n}^{+}(\mathbb{R})$, there are upper triangular matrices $\left[L_{M_{n}}(\mathbf{x})\right],\left[L_{D_{n}}(\mathbf{x})\right]$ and $\left[L_{K_{n}}(\mathbf{x})\right]$ in the set $\mathbb{M}_{n}(\mathbb{R})$ of all the square $(n \times n)$ real matrices, such that

$$
\left[M_{n}(\mathbf{x})\right]=\left[L_{M_{n}}(\mathbf{x})\right]^{T}\left[L_{M_{n}}(\mathbf{x})\right],\left[D_{n}(\mathbf{x})\right]=\left[L_{D_{n}}(\mathbf{x})\right]^{T}\left[L_{D_{n}}(\mathbf{x})\right],\left[K_{n}(\mathbf{x})\right]=\left[L_{K_{n}}(\mathbf{x})\right]^{T}\left[L_{K_{n}}(\mathbf{x})\right] .
$$

As shown by Eq. (4), matrices $\left[M_{n}(\mathbf{x})\right]$ and $\left[K_{n}(\mathbf{x})\right]$ are diagonal but if another projection basis had been chosen these matrices would have been full. Following the construction proposed in Section 2.3.1-(2) and the available information being defined by Eqs. (24), (10) and (25), for all $\mathbf{x}$ in $\mathcal{C}_{\text {par }}$, the random matrices $\left[\mathbf{M}_{n}(\mathbf{x})\right],\left[\mathbf{D}_{n}(\mathbf{x})\right]$ and $\left[\mathbf{K}_{n}(\mathbf{x})\right]$ defined on probability space $\left(\Theta^{\prime}, \mathcal{T}^{\prime}, \mathcal{P}^{\prime}\right)$ are statistically independent and each random matrix belongs to the set $\mathrm{SE}^{+}$ defined in [46]. Consequently, for all $\mathbf{x}$ in $\mathcal{C}_{\text {par }}$, these random matrices are written as

$$
\begin{aligned}
{\left[\mathbf{M}_{n}(\mathbf{x})\right] } & =\left[L_{M_{n}}(\mathbf{x})\right]^{T}\left[\mathbf{G}_{M_{n}}\right]\left[L_{M_{n}}(\mathbf{x})\right], \\
{\left[\mathbf{D}_{n}(\mathbf{x})\right] } & =\left[L_{D_{n}}(\mathbf{x})\right]^{T}\left[\mathbf{G}_{D_{n}}\right]\left[L_{D_{n}}(\mathbf{x})\right], \\
{\left[\mathbf{K}_{n}(\mathbf{x})\right] } & =\left[L_{K_{n}}(\mathbf{x})\right]^{T}\left[\mathbf{G}_{K_{n}}\right]\left[L_{K_{n}}(\mathbf{x})\right],
\end{aligned}
$$

in which the random matrices $\left[\mathbf{G}_{M_{n}}\right],\left[\mathbf{G}_{D_{n}}\right]$ and $\left[\mathbf{G}_{K_{n}}\right]$ are the random germs which are defined on probability space $\left(\Theta^{\prime}, \mathcal{T}^{\prime}, \mathcal{P}^{\prime}\right)$, which are statistically independent and which belong 
to the set $\mathrm{SG}^{+}$defined in $[46]$ and consequently, are random matrices with values in $\mathbf{M}_{n}^{+}(\mathbb{R})$. Since the available information defined by Eq. (25) are inequations, the maximum entropy principle which has been used to construct the probability distribution of the random germs in [41], [42] and [46], introduces one free parameter for each random germ which can be expressed as a function of a dispersion parameter $\delta$ denoting either $\delta_{M}, \delta_{D}$ or $\delta_{K}$ for each one of the three random matrices. Consequently, the probability distributions depend on these dispersion parameters which allow the level of the statistical fluctuations to be controlled. Let $\boldsymbol{\delta}_{\mathbf{G}}=\left(\delta_{M}, \delta_{D}, \delta_{K}\right)$ be the vector of the dispersion parameters belonging to an admissible set $\mathcal{C}_{\mathbf{G}} \subset \mathbb{R}^{3}$. The joint probability density function $p_{\left[\mathbf{G}_{M_{n}}\right],\left[\mathbf{G}_{D_{n}}\right],\left[\mathbf{G}_{K_{n}}\right]}$ of the random matrices $\left[\mathbf{G}_{M_{n}}\right],\left[\mathbf{G}_{D_{n}}\right],\left[\mathbf{G}_{K_{n}}\right]$ is written as

$$
\begin{gathered}
p_{\left[\mathbf{G}_{M_{n}}\right],\left[\mathbf{G}_{D_{n}}\right],\left[\mathbf{G}_{K_{n}}\right]}\left(\left[G_{M_{n}}\right],\left[G_{D_{n}}\right],\left[G_{K_{n}}\right] ; \boldsymbol{\delta}_{\mathbf{G}}\right) \\
=p_{\left.\left[\mathbf{G}_{M_{n}}\right]\right]}\left(\left[G_{M_{n}}\right] ; \delta_{M}\right) \times p_{\left.\left[\mathbf{G}_{D_{n}}\right]\right]}\left(\left[G_{D_{n}}\right] ; \delta_{D}\right) \times p_{\left[\mathbf{G}_{K_{n}}\right]}\left(\left[G_{K_{n}}\right] ; \delta_{K}\right) .
\end{gathered}
$$

The probability distribution of each random matrix $\left[\mathbf{G}_{M_{n}}\right],\left[\mathbf{G}_{D_{n}}\right]$ or $\left[\mathbf{G}_{K_{n}}\right]$ and their algebraic representations useful for generating independent realizations are defined in [46]. Nevertheless, in order that this paper be sufficiently self-contained and in order to simplify its reading, we briefly summarize in the next section the main properties of set $\mathrm{SG}^{+}$.

2.3.5. Ensemble $\mathrm{SG}^{+}$of random matrices. This ensemble is defined as the second-order random matrices $\left[\mathbf{G}_{n}\right]$, defined on the probability space $\left(\Theta^{\prime}, \mathcal{T}^{\prime}, \mathcal{P}^{\prime}\right)$, with values in $\mathbb{M}_{n}^{+}(\mathbb{R})$, such that $E\left\{\left[\mathbf{G}_{n}\right]\right\}=\left[I_{n}\right]$ where $\left[I_{n}\right]$ is the $(n \times n)$ unity matrix and such that $E\left\{\left\|\left[\mathbf{G}_{n}\right]^{-1}\right\|_{F}^{2}\right\}<+\infty$. The level of statistical fluctuations of such a random matrix is controlled by the dispersion parameter $\delta>0$ defined by

$$
\delta=\left\{E\left\{\left\|\left[\mathbf{G}_{n}\right]-\left[I_{n}\right]\right\|_{F}^{2}\right\} /\left\|\left[I_{n}\right]\right\|_{F}^{2}\right\}^{1 / 2}=\left\{E\left\{\left\|\left[\mathbf{G}_{n}\right]-\left[I_{n}\right]\right\|_{F}^{2}\right\} / n\right\}^{1 / 2},
$$

which must be such that $\delta \in\left[0, \delta_{0}\left[\right.\right.$ with $\delta_{0}=(n+1)^{1 / 2}(n+5)^{-1 / 2}$. The probability distribution $P_{\left[\mathbf{G}_{n}\right]}$ of random matrix $\left[\mathbf{G}_{n}\right]$ is completely defined in $[46]$.

The following algebraic representation of the random matrix $\left[\mathbf{G}_{n}\right]$ allows a procedure for the Monte Carlo numerical simulation of random matrix $\left[\mathbf{G}_{n}\right]$ to be defined. The random matrix $\left[\mathbf{G}_{n}\right]$ for which the probability distribution is $P_{\left[\mathbf{G}_{n}\right]}$ can be written as $\left[\mathbf{G}_{n}\right]=\left[\mathbf{L}_{n}\right]^{T}\left[\mathbf{L}_{n}\right]$ in which $\left[\mathbf{L}_{n}\right]$ is an upper triangular random matrix with values in $\mathbf{M}_{n}(\mathbb{R})$ such that: $(1)$ the random variables $\left\{\left[\mathbf{L}_{n}\right]_{j j^{\prime}}, j \leq j^{\prime}\right\}$ are independent; (2) for $j<j^{\prime}$, the real-valued random variable $\left[\mathbf{L}_{n}\right]_{j j^{\prime}}$ can be written as $\left[\mathbf{L}_{n}\right]_{j j^{\prime}}=\sigma_{n} U_{j j^{\prime}}$ in which $\sigma_{n}=\delta(n+1)^{-1 / 2}$ and where $U_{j j^{\prime}}$ is a real-valued Gaussian random variable with zero mean and variance equal to 1 ; (3) for $j=j^{\prime}$, the positive-valued random variable $\left[\mathbf{L}_{n}\right]_{j j}$ can be written as $\left[\mathbf{L}_{n}\right]_{j j}=\sigma_{n} \sqrt{2 V_{j}}$ in which $\sigma_{n}$ is defined above and where $V_{j}$ is a positive-valued gamma random variable whose probability density function $p_{V_{j}}(v)$ with respect to $d v$ is written as $p_{V_{j}}(v)=\mathbb{1}_{\mathbb{R}+}(v)\left\{\Gamma\left(\frac{n+1}{2 \delta^{2}}+\frac{1-j}{2}\right)\right\}^{-1} v^{\frac{n+1}{2 \delta^{2}}-\frac{1+j}{2}} e^{-v}$.

Let $\|[A]\|$ be the operator norm of any matrix $[A]$ belonging to $\mathbb{M}_{n}(\mathbb{R})$ and defined by $\|A\|=\sup _{\|\mathbf{u}\| \leq 1}\|[A] \mathbf{u}\|$ for $\mathbf{u}$ in $\mathbb{R}^{n}$. We have $\|[A] \mathbf{u}\| \leq\|A\|\|\mathbf{u}\|$ for all $\mathbf{u}$ in $\mathbb{R}^{n}$. An important mathematical property of ensemble $\mathrm{SG}^{+}$is the following one. For all $n \geq 2$, we have

$$
E\left\{\left\|\left[\mathbf{G}_{n}\right]^{-1}\right\|^{2}\right\} \leq C_{\delta}<+\infty \quad,
$$

in which $C_{\delta}$ is a positive finite constant that is independent of $n$ but that depends on $\delta$. This inequation means that $n \mapsto E\left\{\left\|\left[\mathbf{G}_{n}\right]^{-1}\right\|^{2}\right\}$ is a bounded function from $\{n \geq 2\}$ into $\mathbb{R}^{+}$. 
2.3.6. Generalized probabilistic model for the random matrices of the stochastic computational model. Taking into account Sections 2.3.1 to 2.3.4, and in particular Eq. (27), the dependent random matrices $\left[\mathbf{M}_{n}(\mathbf{X})\right],\left[\mathbf{D}_{n}(\mathbf{X})\right]$ and $\left[\mathbf{K}_{n}(\mathbf{X})\right]$ introduced in Eq. (14) are written as

$$
\begin{aligned}
{\left[\mathbf{M}_{n}(\mathbf{X})\right] } & =\left[L_{M_{n}}(\mathbf{X})\right]^{T}\left[\mathbf{G}_{M_{n}}\right]\left[L_{M_{n}}(\mathbf{X})\right], \\
{\left[\mathbf{D}_{n}(\mathbf{X})\right] } & =\left[L_{D_{n}}(\mathbf{X})\right]^{T}\left[\mathbf{G}_{D_{n}}\right]\left[L_{D_{n}}(\mathbf{X})\right], \\
{\left[\mathbf{K}_{n}(\mathbf{X})\right] } & =\left[L_{K_{n}}(\mathbf{X})\right]^{T}\left[\mathbf{G}_{K_{n}}\right]\left[L_{K_{n}}(\mathbf{X})\right] .
\end{aligned}
$$

These random matrices which are defined on the probability space $\left(\Theta \times \Theta^{\prime}, \mathcal{T} \otimes \mathcal{T}^{\prime}, \mathcal{P} \otimes \mathcal{P}^{\prime}\right)$ are second-order random variables and their mean values are given by Eq. (12). For all $\theta$ in $\Theta$ and for all $\theta^{\prime}$ in $\Theta^{\prime}$, the realization $\left[\mathbf{M}_{n}\left(\theta^{\prime} ; \mathbf{X}(\theta)\right)\right],\left[\mathbf{D}_{n}\left(\theta^{\prime} ; \mathbf{X}(\theta)\right)\right]$ and $\left[\mathbf{K}_{n}\left(\theta^{\prime} ; \mathbf{X}(\theta)\right)\right]$ of the random matrices $\left[\mathbf{M}_{n}(\mathbf{X})\right],\left[\mathbf{D}_{n}(\mathbf{X})\right]$ and $\left[\mathbf{K}_{n}(\mathbf{X})\right]$ are such that

$$
\begin{aligned}
{\left[\mathbf{M}_{n}\left(\theta^{\prime} ; \mathbf{X}(\theta)\right)\right] } & =\left[L_{M_{n}}(\mathbf{X}(\theta))\right]^{T}\left[\mathbf{G}_{M_{n}}\left(\theta^{\prime}\right)\right]\left[L_{M_{n}}(\mathbf{X}(\theta))\right], \\
{\left[\mathbf{D}_{n}\left(\theta^{\prime} ; \mathbf{X}(\theta)\right)\right] } & =\left[L_{D_{n}}(\mathbf{X}(\theta))\right]^{T}\left[\mathbf{G}_{D_{n}}\left(\theta^{\prime}\right)\right]\left[L_{D_{n}}(\mathbf{X}(\theta))\right], \\
{\left[\mathbf{K}_{n}\left(\theta^{\prime} ; \mathbf{X}(\theta)\right)\right] } & =\left[L_{K_{n}}(\mathbf{X}(\theta))\right]^{T}\left[\mathbf{G}_{K_{n}}\left(\theta^{\prime}\right)\right]\left[L_{K_{n}}(\mathbf{X}(\theta))\right] .
\end{aligned}
$$

The realization $\mathbf{X}(\theta)$ is constructed with a generator of independent realizations of random variable $\mathbf{X}$ whose probability density function $p_{\mathbf{X}}\left(., \boldsymbol{\delta}_{\mathbf{X}}\right)$ is defined by Eq. (23). The independent realizations $\left[\mathbf{G}_{M_{n}}\left(\theta^{\prime}\right)\right],\left[\mathbf{G}_{D_{n}}\left(\theta^{\prime}\right)\right]$ and $\left[\mathbf{G}_{K_{n}}\left(\theta^{\prime}\right)\right]$ are constructed with the generator presented in Section 2.3.5 of random matrix $\left[\mathbf{G}_{n}\right]$ whose probability density function $p_{\left[\mathbf{G}_{n}\right]}\left(\left[G_{n}\right] ; \delta\right)$ is defined in $[46]$.

Let $A$ denoting $M, D$ or $K$ relatively to the generalized mass, damping or stiffness matrix. For all second-order random variable $\mathbf{Q}=\left\{\left(\theta, \theta^{\prime}\right) \mapsto \mathbf{Q}\left(\theta, \theta^{\prime}\right)\right\}$ defined on probability space $\left(\Theta \times \Theta^{\prime}, \mathcal{T} \otimes \mathcal{T}^{\prime}, \mathcal{P} \otimes \mathcal{P}^{\prime}\right)$ with values in $\mathbb{R}^{n}$, it can be proven the following fundamental inequality (non uniform ellipticity condition),

$$
\sqrt{E\left\{<\left[\mathbf{A}_{n}(\mathbf{X})\right] \mathbf{Q}, \mathbf{Q}>^{2}\right\}} \geq c_{A} E\left\{\|\mathbf{Q}\|^{2}\right\},
$$

in which $c_{A}$ is a positive finite real constant independent of dimension $n$. The proof of Eq. (33) is the following. From Eqs. (26) and (32), it can be deduced that

$$
<\left[A_{n}(\mathbf{X}(\theta))\right] \mathbf{Q}\left(\theta, \theta^{\prime}\right), \mathbf{Q}\left(\theta, \theta^{\prime}\right)>\leq\left\|\left[\mathbf{G}_{A_{n}}\left(\theta^{\prime}\right)\right]^{-1}\right\|<\left[\mathbf{A}_{n}\left(\theta^{\prime} ; \mathbf{X}(\theta)\right)\right] \mathbf{Q}\left(\theta, \theta^{\prime}\right), \mathbf{Q}\left(\theta, \theta^{\prime}\right)>.
$$

On the other hand, Eq. (22) yields

$$
\int_{\Theta}<\left[A_{n}(\mathbf{X}(\theta))\right] \mathbf{Q}\left(\theta, \theta^{\prime}\right), \mathbf{Q}\left(\theta, \theta^{\prime}\right)>d \mathcal{P}(\theta) \geq c_{p} \int_{\Theta}\left\|\mathbf{Q}\left(\theta, \theta^{\prime}\right)\right\|^{2} d \mathcal{P}(\theta) .
$$

These two last equations yield

$$
c_{p} E\left\{\|\mathbf{Q}\|^{2}\right\} \leq E\left\{\left\|\left[\mathbf{G}_{A_{n}}\right]^{-1}\right\|<\left[\mathbf{A}_{n}(\mathbf{X})\right] \mathbf{Q}, \mathbf{Q}>\right\} .
$$

Applying the Schwarz inequality to the right-hand side of the above equation and using Eq. (30) yields Eq. (33).

2.3.7. Stochastic solution as a second-order stochastic process. In this section we analyse the time evolution dynamical problem and the associated harmonic problem. 
(i) Stochastic transient solution. Let $T$ be a positive real number. Let us assume that $\int_{0}^{T} E\left\{\left\|\mathbf{f}^{n}(t ; \mathbf{X})\right\|^{2} d t<+\infty\right.$. Using a similar demonstration that the one given in [42] and taking into account the property defined by Eq. (33), it can be proven that, for $t$ in $] 0, T]$ and for zero initial conditions, the stochastic solution of Eqs. (13) and (14) is a unique second-order stochastic process. This means that for all $t$ fixed in $[0, T]$, we have

$$
E\left\{\left\|\mathbf{Y}^{n}(t)\right\|^{2}\right\} \leq C_{1}<+\infty \quad, \quad E\left\{\left\|\dot{\mathbf{Y}}^{n}(t)\right\|^{2}\right\} \leq C_{2}<+\infty \quad,
$$

in which $C_{1}$ and $C_{2}$ are positive constants that are independent of $n$ and $t$ but which depend on the dispersion parameters $\boldsymbol{\delta}_{\mathbf{X}}$ and $\boldsymbol{\delta}_{\mathbf{G}}$.

(ii) Stochastic solution in the frequency domain. Let $\mathbf{u}(\omega)=\int_{\mathbb{R}} e^{-i \omega t} \mathbf{u}(t) d t$ be the Fourier transform of the time function $t \mapsto \mathbf{u}(t)$. The harmonic problem associated with Eq. (1) is, for all frequency $\omega$ belonging to the frequency band of analysis is $\left.B=] 0, \omega_{\max }\right]$, written as

$$
\left(-\omega^{2}[\mathbb{M}(\mathbf{x})]+i \omega[\mathbb{D}(\mathbf{x})]+[\mathbb{K}(\mathbf{x})]\right) \mathbf{y}(\omega)=\mathbf{f}(\omega ; \mathbf{x}) \quad .
$$

Consequently, the stochastic time evolution dynamical problem defined by Eqs. (13) and (14) is replaced by the following stochastic harmonic dynamical problem. For all $\omega$ in $B$, construct the $\mathbb{C}^{m}$-valued random variable $\mathbf{Y}^{n}(\omega)$ such that

$$
\begin{gathered}
\mathbf{Y}^{n}(\omega)=\left[\phi_{n}(\mathbf{X})\right] \mathbf{Q}^{n}(\omega), \\
\left(-\omega^{2}\left[\mathbf{M}_{n}(\mathbf{X})\right]+i \omega\left[\mathbf{D}_{n}(\mathbf{X})\right]+\left[\mathbf{K}_{n}(\mathbf{X})\right]\right) \mathbf{Q}^{n}(\omega)=\mathbf{f}^{n}(\omega ; \mathbf{X}),
\end{gathered}
$$

in which $\mathbf{f}^{n}(\omega ; \mathbf{X})=\left[\phi_{n}(\mathbf{X})\right]^{T} \mathbf{f}(\omega ; \mathbf{X})$ and where $\mathbf{Y}^{n}(\omega)=\left\{\left(\theta, \theta^{\prime}\right) \mapsto \mathbf{Y}^{n}\left(\theta, \theta^{\prime} ; \omega\right)\right\}$ is a $\mathbb{C}^{m}$ valued random vector and $\mathbf{Q}^{n}(\omega)=\left\{\left(\theta, \theta^{\prime}\right) \mapsto \mathbf{Q}^{n}\left(\theta, \theta^{\prime} ; \omega\right)\right\}$ is an $\mathbb{C}^{n}$-valued random vector defined on probability space $\left(\Theta \times \Theta^{\prime}, \mathcal{T} \otimes \mathcal{T}^{\prime}, \mathcal{P} \otimes \mathcal{P}^{\prime}\right)$. As for the stochastic transient solution, it can be proven that, for all $\omega$ in $B$, the stochastic harmonic problem defined by Eqs. (36) and (37) has a unique second-order stochastic solution such that

$$
E\left\{\left\|\mathbf{Y}^{n}(\omega)\right\|^{2}\right\} \leq C<+\infty \quad,
$$

in which $C$ is a positive constant that is independent of $n$ and $\omega$ but which depends on the dispersion parameters $\boldsymbol{\delta}_{\mathbf{X}}$ and $\boldsymbol{\delta}_{\mathbf{G}}$. For the numerical Monte Carlo simulation, Eqs. (15) and (16) must be replaced by the following one,

$$
\begin{gathered}
\mathbf{Y}^{n}\left(\theta, \theta^{\prime} ; \omega\right)=\left[\phi_{n}(\mathbf{X}(\theta))\right] \mathbf{Q}^{n}\left(\theta, \theta^{\prime} ; \omega\right), \\
\left(-\omega^{2}\left[\mathbf{M}_{n}\left(\theta^{\prime} ; \mathbf{X}(\theta)\right)\right]+i \omega\left[\mathbf{D}_{n}\left(\theta^{\prime} ; \mathbf{X}(\theta)\right)\right]+\left[\mathbf{K}_{n}\left(\theta^{\prime} ; \mathbf{X}(\theta)\right)\right]\right) \mathbf{Q}^{n}\left(\theta, \theta^{\prime} ; \omega\right)=\mathbf{f}^{n}(\omega ; \mathbf{X}(\theta)) .
\end{gathered}
$$

3. Estimation of the dispersion parameters of the prior probability models of the generalized probabilistic approach of uncertainties

In Section 2, we have presented a formulation for a prior generalized probabilistic approach of uncertainties allowing model-parameter uncertainties and model uncertainties (modeling errors) to be separately taken into account in the computational model. Firstly, this formulation introduces a prior probability model $p_{\mathbf{X}}\left(\mathbf{x} ; \boldsymbol{\delta}_{\mathbf{X}}\right)$ of random variable $\mathbf{X}$ relative to 
the model-parameter uncertainties and depending of a free dispersion parameter $\boldsymbol{\delta}_{\mathbf{X}}$ belonging to an admissible set $\mathcal{C}_{\mathbf{X}}$ which is a subset of $\mathbb{R}^{\mu}$. Secondly, this formulation introduces a prior probability model $p_{\left[\mathbf{G}_{\left.M_{n}\right]}\right]}\left(\left[G_{M_{n}}\right] ; \delta_{M}\right), p_{\left.\left[\mathbf{G}_{D_{n}}\right]\right]}\left(\left[G_{D_{n}}\right] ; \delta_{D}\right)$ and $p_{\left.\left[\mathbf{G}_{K_{n}}\right]\right]}\left(\left[G_{K_{n}}\right] ; \delta_{K}\right)$ of independent random matrices $\left.\left.\left[\mathbf{G}_{M_{n}}\right]\right],\left[\mathbf{G}_{D_{n}}\right]\right]$ and $\left.\left[\mathbf{G}_{K_{n}}\right]\right]$ relative to the model uncertainties (modeling errors) and depending on a free dispersion parameter $\boldsymbol{\delta}_{\mathbf{G}}=\left(\delta_{M}, \delta_{D}, \delta_{K}\right)$ belonging to an admissible set $\mathcal{C}_{\mathbf{G}}$ which is a subset of $\mathbb{R}^{3}$.

If no experimental data are available, then the dispersions parameters $\boldsymbol{\delta}_{\mathbf{X}}$ and $\boldsymbol{\delta}_{\mathbf{G}}$ must be considered as parameters to perform a sensitivity analysis of the stochastic solution. Such a prior generalized probabilistic approach of uncertainties then allows the robustness of the solution to be analyzed in function of the level of model-parameter uncertainties controlled by $\boldsymbol{\delta}_{\mathbf{X}}$ and of the level of model uncertainties (modeling errors) controlled by $\boldsymbol{\delta}_{\mathbf{G}}$.

For the particular case for which a few experimental data exist, we propose a methodology to estimate the dispersion parameters of the prior probability models of uncertainties.

\subsection{Estimation of the dispersion parameter of the prior probability model of the uncertain model parameter}

The first step of the method proposed consists in estimating the dispersion parameter $\boldsymbol{\delta}_{\mathbf{X}} \in \mathcal{C}_{\mathbf{X}} \subset \mathbb{R}^{\mu}$ of the prior probability model of the uncertain model parameter in considering that there is no modeling error and consequently in using the stochastic computational model with $\boldsymbol{\delta}_{\mathbf{G}}=0$ (no modeling errors). The estimation of $\boldsymbol{\delta}_{\mathbf{X}}$ must then be performed with observations of the systems which are weakly sensitive to modeling errors and for which experimental data are available. There are several possibilities in the choice of such observations satisfying these criteria. Nevertheless, in order to limit the developments, we will propose only one of the possibilities which is in the framework of experimental modal analysis. Note that for a complex dynamical system, the first eigenfrequencies and the associated elastic modes can be experimentally measured. In addition, if the corresponding computational model is sufficiently large, the first eigenfrequencies predicted with the computational model depend on the model-parameter uncertainties but must not depend on modeling errors (if the fundamental eigenfrequency of the mean computational model cannot reasonably be predicted due to the presence of significant model errors, this means that the mean computational model cannot be considered as a correct model and must be rebuilt).

Let us assumed that the first $\widetilde{\mu}$ experimental eigenfrequencies $\nu_{1}^{\text {ref }}, \ldots, \nu_{\widetilde{\mu}}^{\text {ref }}$ are measured. Let $\lambda_{\alpha}^{\text {ref }}=\left(2 \pi \nu_{\alpha}^{\text {ref }}\right)^{2}$ be the corresponding experimental eigenvalues. Let $\Lambda_{1}, \ldots, \Lambda_{\widetilde{\mu}}$ be the corresponding eigenvalues of this stochastic computational model. Let $p_{\Lambda_{1}, \ldots, \Lambda_{\tilde{\mu}}}\left(\lambda_{1}, \ldots, \lambda_{\widetilde{\mu}} ; \boldsymbol{\delta}_{\mathbf{X}}\right)$ be the joint probability density function of the random variables $\Lambda_{1}, \ldots, \Lambda_{\widetilde{\mu}}$. The optimal value $\boldsymbol{\delta}_{\mathbf{X}}^{\text {opt }}$ of the dispersion parameter $\boldsymbol{\delta}_{\mathbf{X}}$ can be estimated by maximizing the Neperian logarithm of the likelihood function (maximum likelihood method [39],[52]),

$$
\boldsymbol{\delta}_{\mathbf{X}}^{\text {opt }}=\arg \max _{\boldsymbol{\delta}_{\mathbf{X}} \in \mathcal{C}_{\mathbf{X}}}\left\{\ln \left(p_{\Lambda_{1}, \ldots, \Lambda_{\widetilde{\mu}}}\left(\lambda_{1}^{\mathrm{ref}}, \ldots, \lambda_{\widetilde{\mu}}^{\mathrm{ref}} ; \boldsymbol{\delta}_{\mathbf{X}}\right)\right)\right\}
$$

in which $p_{\Lambda_{1}, \ldots, \Lambda_{\widetilde{\mu}}}\left(\lambda_{1}^{\mathrm{ref}}, \ldots, \lambda_{\widetilde{\mu}}^{\mathrm{ref}} ; \boldsymbol{\delta}_{\mathbf{X}}\right)$ is estimated using the stochastic computational model with $\boldsymbol{\delta}_{\mathbf{G}}=0$ and which is solved by the Monte Carlo numerical method. 


\subsection{Estimation of the dispersion parameters of the prior probability model of model uncertainties}

The second step of the method proposed consists in estimating the dispersion parameter $\boldsymbol{\delta}_{\mathbf{G}} \in \mathcal{C}_{\mathbf{G}} \subset \mathbb{R}^{3}$ of the prior probability model of the model uncertainties (modeling errors) in presence of the model-parameter uncertainties estimated in the first step that is to say in using the stochastic computational model with $\boldsymbol{\delta}_{\mathbf{X}}=\boldsymbol{\delta}_{\mathbf{x}}^{\text {opt }}$. For such an estimation, it is assumed that one experimental frequency response function is available for one or for a few observation points. We then consider the stochastic reduced computational model in the frequency domain defined by Eqs. (36) and (37). It is assumed that $n_{\text {obs }}$ observations are introduced and are such that $\mathbf{Y}^{\text {obs }}(\omega)=\left(\left|Y_{j_{1}}^{n}(\omega)\right|, \ldots,\left|Y_{j_{n_{o b s}}}^{n}(\omega)\right|\right)$ are observed in $n_{f r e q}$ frequencies $\omega_{1}, \ldots, \omega_{n_{\text {freq }}}$ in the frequency band of analysis $B$. We then introduce the random observed vector $\mathbb{Y}=\left(\mathbf{Y}^{\text {obs }}\left(\omega_{1}\right), \ldots, \mathbf{Y}^{\text {obs }}\left(\omega_{n_{\text {freq }}}\right)\right)$ with values in $\mathbb{R}^{s}$ with $s=n_{\text {obs }} \times n_{f r e q}$. Let $y^{\text {ref }}$ be the deterministic vector in $\mathbb{R}^{s}$ made up of the experimental data and corresponding to $\mathbb{R}^{s}$-valued random vector $\mathbb{Y}$ of the stochastic computational model. The estimation of $\boldsymbol{\delta}_{\mathbf{G}}$ can be performed by using the maximum likelihood method. Since $s$ can be very high, this method requires a big computational effort. We then propose to perform a usual statistical reduced representation (representation similar to the principal component analysis or Karhunen-Loeve expansion) and then to apply the maximum likelihood method to such a reduced representation $[50]$.

For $\boldsymbol{\delta}_{\mathbf{X}}=\boldsymbol{\delta}_{\mathbf{X}}^{\text {opt }}$ and for each fixed value of $\boldsymbol{\delta}_{\mathbf{G}}$, let $\mathbf{m}_{\mathbf{Y}}\left(\boldsymbol{\delta}_{\mathbf{G}}\right)$ be the $\mathbb{R}^{s}$-valued mean vector and let $\left[C_{\mathbb{Y}}\left(\boldsymbol{\delta}_{\mathbf{G}}\right)\right]$ be the $(s \times s)$ covariance matrix of the random vector $\mathbb{Y}$ computed with the stochastic reduced model and defined by

$$
\mathrm{m}_{\mathbb{Y}}\left(\boldsymbol{\delta}_{\mathbf{G}}\right)=E\{\mathbb{Y}\} \quad, \quad\left[C_{\mathbb{Y}}\left(\boldsymbol{\delta}_{\mathbf{G}}\right)\right]=E\left\{\left(\mathbb{Y}-\mathrm{m}_{\mathbb{Y}}\left(\boldsymbol{\delta}_{\mathbf{G}}\right)\right)\left(\mathbb{Y}-\mathrm{m}_{\mathbb{Y}}\left(\boldsymbol{\delta}_{\mathbf{G}}\right)\right)^{T}\right\} \quad .
$$

For each given value of $\boldsymbol{\delta}_{\mathbf{G}}, \mathrm{m}_{\mathbb{Y}}\left(\boldsymbol{\delta}_{\mathbf{G}}\right)$ and $\left[C_{\mathbb{Y}}\left(\boldsymbol{\delta}_{\mathbf{G}}\right)\right]$ can easily be estimated by using the stochastic computational model with the Monte Carlo method. We introduce the eigenvalue problem

$$
\left[C_{\mathbb{Y}}\left(\boldsymbol{\delta}_{\mathbf{G}}\right)\right] \gtrless\left(\boldsymbol{\delta}_{\mathbf{G}}\right)=\lambda\left(\boldsymbol{\delta}_{\mathbf{G}}\right) \gtrless\left(\boldsymbol{\delta}_{\mathbf{G}}\right),
$$

for which the positive eigenvalues are such that $\lambda_{1}\left(\boldsymbol{\delta}_{\mathbf{G}}\right) \geq \lambda_{2}\left(\boldsymbol{\delta}_{\mathbf{G}}\right) \geq \ldots$. The corresponding eigenvectors $\mathrm{x}^{1}\left(\boldsymbol{\delta}_{\mathbf{G}}\right), \mathrm{k}^{2}\left(\boldsymbol{\delta}_{\mathbf{G}}\right), \ldots$ belong to $\mathbb{R}^{s}$ and constitute an orthonormal family of vectors for the Euclidean norm. Let $\mu^{\prime}$ be an integer such that $1 \leq \mu^{\prime}<s$. We introduce the approximation $\Upsilon^{\mu^{\prime}}$ of $Y$ defined by

$$
\mathbb{Y}^{\mu^{\prime}}=\mathrm{m}_{\mathbb{Y}}\left(\boldsymbol{\delta}_{\mathbf{G}}\right)+\sum_{j=1}^{\mu^{\prime}} \sqrt{\lambda_{j}\left(\boldsymbol{\delta}_{\mathbf{G}}\right)} Z_{j} \mathfrak{R}^{j}\left(\boldsymbol{\delta}_{\mathbf{G}}\right)
$$

in which $\mathbf{Z}=\left(Z_{1}, \ldots, Z_{\mu^{\prime}}\right)$ is a $\mathbb{R}^{\mu^{\prime}}$-valued random variable such that

$$
Z_{j}=\frac{1}{\sqrt{\lambda_{j}\left(\boldsymbol{\delta}_{\mathbf{G}}\right)}}<\mathbb{Y}-\mathrm{m}_{\mathbb{Y}}\left(\boldsymbol{\delta}_{\mathbf{G}}\right), \mathfrak{Z}^{j}\left(\boldsymbol{\delta}_{\mathbf{G}}\right)>\quad .
$$

It is known that the components of $\mathbf{Z}$ are second-order centered random variables which are uncorrelated, that is to say, are such that

$$
E\left\{\mathbf{Z} \mathbf{Z}^{T}\right\}=\left[I_{\mu^{\prime}}\right] .
$$


The order $\mu^{\prime}$ of the statistical reduction is calculated in order to get an approximation with a given accuracy $\varepsilon$, independent of $\mu^{\prime}$ and $\boldsymbol{\delta}_{\mathbf{G}}$, such that, for all $\boldsymbol{\delta}_{\mathbf{G}}$ in $\mathcal{C}_{\mathbf{G}}$,

$$
E\left\{\left\|Y-\Psi^{\mu^{\prime}}\right\|^{2}\right\}=\varepsilon E\left\{\|\bigvee\|^{2}\right\}
$$

Consequently, $\mu^{\prime}$ must be chosen such that

$$
\operatorname{criter}_{\Upsilon}\left(\mu^{\prime}\right) \leq \varepsilon \quad, \quad \operatorname{criter}_{\Upsilon}\left(\mu^{\prime}\right)=\max _{\boldsymbol{\delta}_{\mathbf{G}} \in \mathcal{C}_{\mathbf{G}}}\left(1-\frac{\sum_{j=1}^{\mu^{\prime}} \lambda_{j}\left(\boldsymbol{\delta}_{\mathbf{G}}\right)}{\operatorname{tr}\left[C_{\Im}\left(\boldsymbol{\delta}_{\mathbf{G}}\right)\right]}\right),
$$

in which $\operatorname{tr}$ is the trace of matrix. In practice, for $\boldsymbol{\delta}_{\mathbf{G}}$ given in $\mathcal{C}_{\mathbf{G}}$, the dominant subspace of matrix $\left[C_{\mathbb{Y}}\left(\boldsymbol{\delta}_{\mathbf{G}}\right)\right]$ associated with the $\mu^{\prime}$ first largest eigenvalues $\lambda_{1}\left(\boldsymbol{\delta}_{\mathbf{G}}\right) \geq \ldots \geq \lambda_{\mu^{\prime}}\left(\boldsymbol{\delta}_{\mathbf{G}}\right)$ is calculated by using an iteration algorithm such as the subspace iteration method. The statistical reduction will be efficient if $\mu^{\prime} \ll s$. Let $\mathbf{z}^{\mathrm{ref}}\left(\boldsymbol{\delta}_{\mathbf{G}}\right)=\left(z_{1}^{\mathrm{ref}}\left(\boldsymbol{\delta}_{\mathbf{G}}\right), \ldots, z_{\mu^{\prime}}^{\mathrm{ref}}\left(\boldsymbol{\delta}_{\mathbf{G}}\right)\right.$ be the vector in $\mathbb{R}^{\mu^{\prime}}$ corresponding to $\mathbf{Z}$ for the experimental data such that

$$
z_{j}^{\mathrm{ref}}\left(\boldsymbol{\delta}_{\mathbf{G}}\right)=\frac{1}{\sqrt{\lambda_{j}\left(\boldsymbol{\delta}_{\mathbf{G}}\right)}}<\mathrm{y}^{\mathrm{ref}}-\mathrm{m}_{\mathbb{Y}}\left(\boldsymbol{\delta}_{\mathbf{G}}\right), \mathbb{Z}^{j}\left(\boldsymbol{\delta}_{\mathbf{G}}\right)>\quad .
$$

The estimation of $\boldsymbol{\delta}_{\mathbf{G}}$ is performed using the maximum likelihood method for the random vector $\mathbf{Z}=\left(Z_{1}, \ldots, Z_{\mu^{\prime}}\right)$ whose components are centered and uncorrelated (but dependent) random variables. The Neperian logarithm of the likelihood function is then defined by

$$
\mathcal{L}\left(\boldsymbol{\delta}_{\mathbf{G}}\right)=\sum_{j=1}^{\mu^{\prime}}\left\{\ln \left(p_{Z_{j}}\left(z_{j}^{\mathrm{ref}}\left(\boldsymbol{\delta}_{\mathbf{G}}\right) ; \boldsymbol{\delta}_{\mathbf{X}}^{\text {opt }}, \boldsymbol{\delta}_{\mathbf{G}}\right)\right\}\right.
$$

in which, for all $j$ in $\left\{1, \ldots \mu^{\prime}\right\}$, the probability density function $z \mapsto p_{Z_{j}}\left(z ; \boldsymbol{\delta}_{\mathbf{X}}^{\text {opt }}, \boldsymbol{\delta}_{\mathbf{G}}\right)$ depends on the known parameter $\boldsymbol{\delta}_{\mathbf{X}}^{\text {opt }}$ and on the unknown parameter $\boldsymbol{\delta}_{\mathbf{G}}$ which must be estimated. This likelihood function is estimated with the stochastic reduced model. The optimal value $\boldsymbol{\delta}_{\mathbf{G}}^{\text {opt }}$ of $\boldsymbol{\delta}_{\mathbf{G}}$ is then given as the solution of the following optimization problem,

$$
\boldsymbol{\delta}_{\mathbf{G}}^{\text {opt }}=\arg \max _{\boldsymbol{\delta}_{\mathbf{G}} \in \mathcal{C}_{\mathbf{G}}} \mathcal{L}\left(\boldsymbol{\delta}_{\mathbf{G}}\right)
$$

4. Validation of the prior generalized probabilistic approach of uncertainties

In this Section, we present a validation of the previous theory for which the real system corresponds to the linear elastodynamics of a three-dimensional slender elastic bounded medium for which the mean model is carried out using the Euler beam theory. The question analyzed in this section is not to decide if this choice is good or bad but, assuming that the mean model is constructed using the Euler beam theory, to see how model-parameter uncertainties and modeling errors can separately be identified using a given reference solution relative to the real system. 


\subsection{Designed system}

The designed system is a slender cylindrical elastic medium defined in a cartesian co-ordinate system $\left(O x_{1} x_{2} x_{3}\right)$. The cylinder has length $L_{1}=10 \mathrm{~m}$ and has a rectangular section with height $L_{2}=1.1 \mathrm{~m}$ and width $L_{3}=1.6 \mathrm{~m}$. The two end sections are located at $x_{1}=0$ and $x_{1}=10$. The origine $O$ is in the corner of the end section and $O x_{1}$ is parallel to the cylinder axis. Axis $O x_{2}$ is the transversal axis along the height and $O x_{3}$ is the lateral axis along the width. The neutral line has for equation $\left\{0 \leq x_{1} \leq 10 ; x_{2}=0.550 ; x_{3}=0.800\right\}$. The elastic medium is made of a composite material. Concerning the boundary conditions, the displacement field is zero on the part of the boundary defined by $\left\{\left(x_{1}, x_{2}, x_{3}\right): x_{1}=0 ; 0 \leq x_{2} \leq 0.11 ; 0 \leq x_{3} \leq\right.$ $1.6\}$ and by $\left\{\left(x_{1}, x_{2}, x_{3}\right): x_{1}=10 ; 0 \leq x_{2} \leq 0.11 ; 0 \leq x_{3} \leq 1.6\right\}$. The other parts of the boundary of the domain are free. This means that the structure is almost simply supported on the two lines defined by $\left\{x_{1}=0 ; x_{2}=0 ; 0 \leq x_{3} \leq 1.6\right\}$ and $\left\{x_{2}=10 ; x_{2}=0 ; 0 \leq x_{3} \leq 1.6\right\}$. The frequency band of analysis is $B=] 0,1200] \mathrm{Hz}$. The external load is a point load applied at the point $\left(x_{1}=4.250, x_{2}=1.100, x_{3}=0.800\right)$ and its Fourier transform is the vector-valued function $\nu \mapsto\left(0,-\mathbb{1}_{B}(\nu), 0\right)$ in which $\mathbb{1}_{B}(\nu)=1$ if frequency $\nu$ belongs to $B$ and $\mathbb{1}_{B}(\nu)=0$ if $\nu$ does not belong to $B$. We are interested in the transversal displacement along $O x_{2}$ in the plane $O x_{1} x_{2}$ of the neutral line at the six observation points $P_{1}, P_{2}, P_{3}, P_{4}, P_{5}$ and $P_{6}$ belonging to the neutral line for which $x_{1}$ are $1.875,3.125,4.250,5.000,6.375$ and $9.250 m$ respectively.

\subsection{Real system and generation of a reference response by numerical simulation}

In this application, no experimental results are available. Consequently, a reference solution of the real system has been constructed (1) in developing a 3D elastic model of the real system defined in Section 4.1, (2) in discretizing this model by the finite element method and (3) in solving the equation in the frequency domain with a frequency resolution of $1 \mathrm{~Hz}$. In the model, the material is taken as homogeneous and isotropic with a Young modulus $1.1 \times 10^{10} \mathrm{~N} / \mathrm{m}^{2}$, a Poisson coefficient 0.17 and a mass density $1600 \mathrm{Kg} / \mathrm{m}^{3}$. The damping rate is 0.01 . The finite element mesh is made up of $80 \times 10 \times 14=11,200$ threedimensional 8-nodes solid elements. There are 13,365 nodes and a total of 39,915 degrees of freedom (due to the boundary conditions, the displacement is zero for $2 \times 30$ nodes). There are 144 eigenfrequencies in band ]0,1200] $\mathrm{Hz}$ and 39 eigenfrequencies in frequency band $] 1200,1300] \mathrm{Hz}$. The fundamental eigenfrequency is $\nu_{1}^{\text {ref }}=18.8 \mathrm{~Hz}$. There are 13 eigenfrequencies in frequency band $] 0,200] \mathrm{Hz}$. The eigenfrequencies of the first 4 bending modes corresponding to the first 4 elastic modes having respectively 2 to 5 nodes (zero $O x_{2}$ displacement) on the neutral line are $\nu_{j_{1}}^{\text {ref }}=18.8, \nu_{j_{2}}^{\text {ref }}=45.4, \nu_{j_{3}}^{\text {ref }}=103.8, \nu_{j_{4}}^{\text {ref }}=170.5 H z$ with $j_{1}=1, j_{2}=3, j_{3}=7, j_{4}=10$.

\subsection{Mean model prediction and comparison with the reference response}

The mean model, as the predictive model of the real system defined in Section 4.2, is constructed from the designed system defined in Section 4.1. This mean model is made up of a damped homogeneous Euler elastic beam with length $L_{1}=10 \mathrm{~m}$, simply supported at $x_{1}=0$ and $x_{1}=10$. The material is taken as homogeneous. The bending stiffness is then $\underline{k}=3.68953 \times 10^{9} \mathrm{~N} \times \mathrm{m}^{2}$ and the linear mass density is $\underline{\rho}_{1}=2816 \mathrm{Kg} / \mathrm{m}$. We then have $\underline{k} / \underline{\rho}_{1}=1.3102 \times 10^{6} \mathrm{~m}^{4} / \mathrm{s}^{2}$. Let $\underline{x}$ be such that $\underline{k} / \underline{\rho}_{1}=\underline{x} L_{2}^{2} / 12=0.10083 \underline{x}$ which yields $\underline{x}=1.299375 \times 10^{7} \mathrm{~m}^{2} / \mathrm{s}^{2}$. The damping rate is 0.01 . This mean model is used to predict the 
transversal displacement (following $O x_{2}$ ) corresponding to the bending vibrations in the plane $\left(O x_{1} x_{2}\right)$. Observation point $P_{3}$ corresponds to the driven point (excitation point). There are 8 eigenfrequencies in band $B$ and 0 eigenfrequency in frequency band $[1200,1300] \mathrm{Hz}$. The fundamental eigenfrequency is $\nu_{1}=17.98 \mathrm{~Hz}$. The eigenfrequencies of the first 4 bending modes corresponding to the first 4 elastic modes having respectively 2 to 5 nodes (zero $O x_{2^{-}}$ displacement) on the neutral line are $\nu_{1}=17.98, \nu_{2}=71.9, \nu_{3}=161.8, \nu_{4}=287.7 \mathrm{~Hz}$. The frequency response functions at the 6 observation points are calculated using modal analysis with the first 80 elastic modes over the frequency band $B$ with a frequency resolution of $1 \mathrm{~Hz}$.
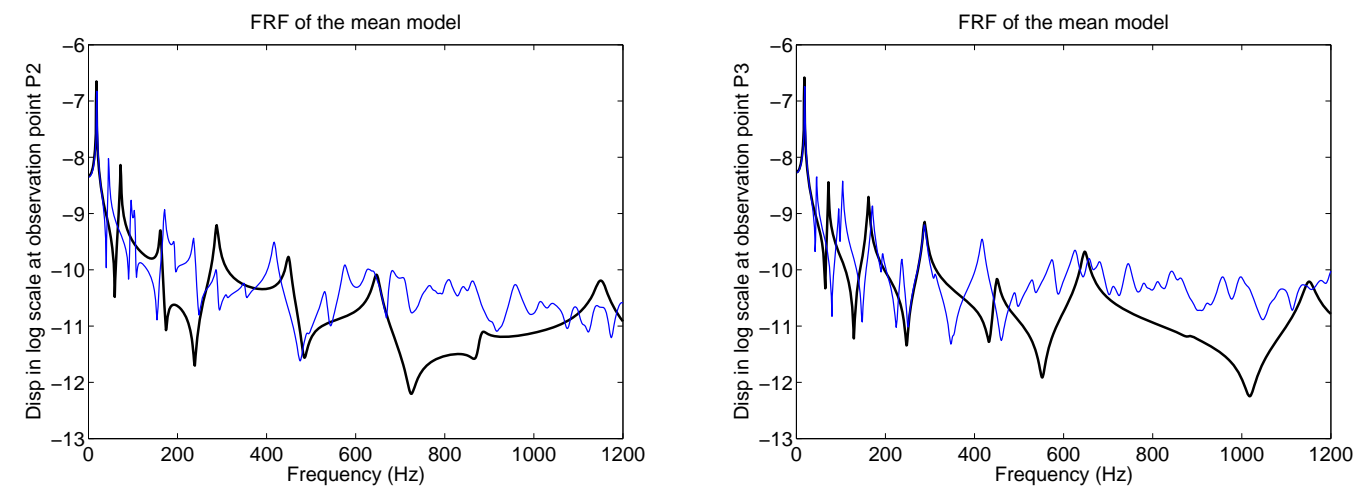

Figure 1. Frequency response function for transversal displacement of the neutral line: reference response (thin solid line), mean model (thick solid line). Observations $P_{2}$ (left) and $P_{3}$ (right).
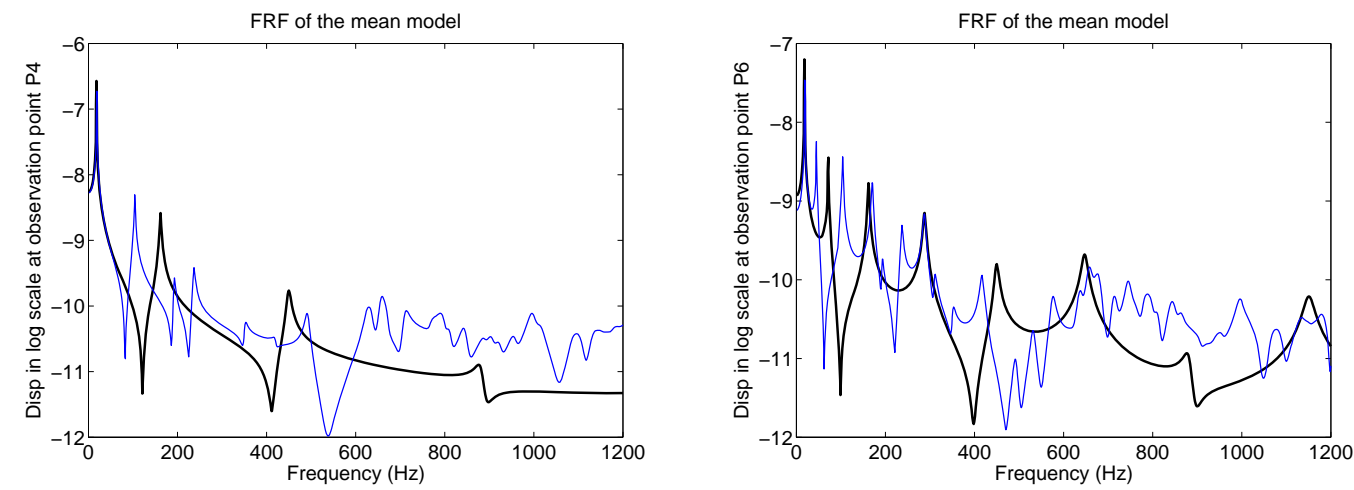

Figure 2. Frequency response function for transversal displacement of the neutral line: reference response (thin solid line), mean model (thick solid line). Observations $P_{4}$ (left) and $P_{6}$ (right).

Figure 1 (left and right) and Figure 2 (left and right) display the frequency response functions for the transversal displacements at observation points $P_{2}, P_{3}, P_{4}$ and $P_{6}$ of the neutral line. The mean model prediction is compared to the reference solution. It can be seen, that there are significant differences which are due to the boundary conditions which are not correctly modeled by a simply supported beam but above all, which are due to the presence of elastic 
modes in the real system (induced by the lateral dimension) which cannot be predicted with the Euler beam theory. This means that there are model-parameter uncertainties but above all there are modeling errors.

\subsection{Estimation of the dispersion parameter of the prior probability model of the uncertain model parameter}

As we have explained in Sections 3 and 4.3, there are two types of uncertainties. In this section, the model-parameter uncertainties of the mean model are taken into account using the parametric probabilistic approach. We used the method presented in Section 3.1. From Sections 4.3 and 4.2 , it can be seen that the fundamental eigenfrequency of the mean model is $\nu_{1}=17.98 \mathrm{~Hz}$ which must be compared to the reference fundamental frequency $\nu_{1}^{\text {ref }}=18.8 \mathrm{~Hz}$. The difference between these two frequencies is then due to model-parameter uncertainties. Let $\lambda_{1}=\left(2 \pi \nu_{1}\right)^{2}=12,762$ be the lowest eigenvalue associated with the fundamental eigenfrequency of the mean model. From the Euler beam theory, we can write $\lambda_{1}=a_{1} \underline{x}$ with $a_{1}=9.8216 \times 10^{-4}$. Therefore, $\underline{x}$ is modeled by a random variable $X$ and consequently, $\lambda_{1}$ becomes a random variable $\Lambda_{1}$ such that $\Lambda_{1}=a_{1} X$. The corresponding lowest eigenvalue of the real system is then $\lambda_{1}^{\text {ref }}=13,953$.

In order to construct the prior probability model of random variable $X$ using Information Theory (see Section 2.3.3, we need to identify the available information of $X$ which is the following: (i) $X$ is a random variable with values in $\left.\mathbb{R}^{+}=\right] 0+\infty$. (ii) The mean value of $X$ is $\underline{x}$ which means that $E\{X\}=\underline{x}$. (iii) $X^{-1}$ must be a second-order random variable, i.e. $E\left\{X^{-2}\right\}<+\infty$ which is satisfied if $E\{\ln (X)\}=v$ with $|v|<+\infty$ imposed.

The unknown prior probability density function $x \mapsto p_{X}(x)$ on $\mathbb{R}$ with support $\mathbb{R}^{+}$of random variable $X$ (prior probability density function) is then constructed by using the maximum entropy principle under the constraints defined by the available information (i), (ii) and (iii). This probability density function depends on the arbitrary constant $v$ which does not have any physical meaning. In this condition, constant $v$ is expressed in function of the dispersion parameter $\delta_{X}=\sigma_{X} / \underline{x}$ (coefficient of variation of $X$ in which $\sigma_{X}$ is the standard deviation of $X$ ) which allows the level of statistical fluctuations of $X$ to be controlled and yields [46] a Gamma density probability density function which can be written for $0 \leq \delta_{X}<1 / \sqrt{2}$ as

$$
p_{X}(x)=\mathbb{1}_{] 0,+\infty[}(x) \frac{1}{\underline{x}} \frac{\delta_{X}^{-2 \delta_{X}^{-2}}}{\Gamma\left(\delta_{X}^{-2}\right)}(x / \underline{x})^{\left(\delta_{X}^{-2}-1\right)} \exp \left(-\frac{(x / \underline{x})}{\delta_{X}^{2}}\right),
$$

where $\Gamma(\alpha)=\int_{0}^{+\infty} t^{\alpha-1} e^{-t} d t$ is the Gamma function.

The identification of parameter $\delta_{X}$ is performed as explained in Section 3.1 using the maximum likelihood method for the lowest random eigenvalue $\Lambda_{1}$ for which its probability density function $\lambda \mapsto p_{\Lambda_{1}}\left(\lambda ; \delta_{X}\right)$ depends on parameter $\delta_{X}$ and which can be constructed with the stochastic model. There is only one known "experimental value" with is the reference value $\lambda_{1}^{\text {ref }}$. The optimal value $\delta_{X}^{\text {opt }}$ is then given by maximizing the Neperian logarithm of the likelihood function (see Eq. (41)),

$$
\delta_{X}^{\text {opt }}=\arg \max _{\delta_{X}}\left\{\ln \left(p_{\Lambda_{1}}\left(\lambda_{1}^{\text {ref }} ; \delta_{X}\right)\right)\right\} .
$$


Figure 3 displays the graph of function $\delta_{X} \mapsto \ln \left(p_{\Lambda_{1}}\left(\lambda_{1}^{\text {ref }} ; \delta_{X}\right)\right)$ and the optimal value is $\delta_{X}^{\text {opt }}=0.093$.

We consider the stochastic reduced model constructed with the parametric probabilistic

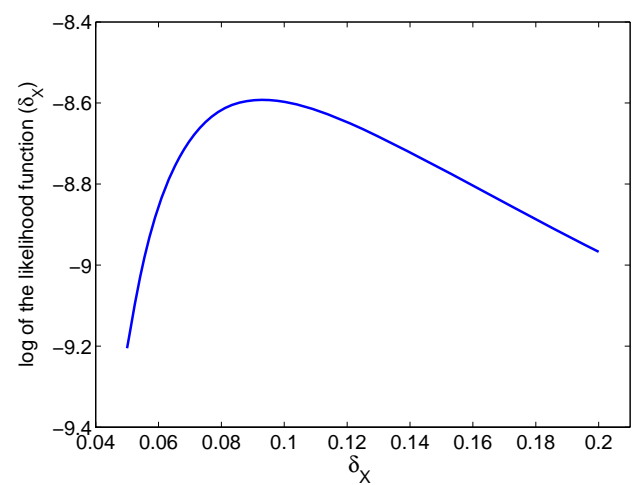

Figure 3. Graph of the Neperian logarithm of likelihood function as a function of the dispersion parameter $\delta_{X}$ for the identification of the probabilistic model of the uncertain parameter $X$.

approach of uncertain parameter $X$ for which the probabilistic model is defined above with $\delta_{X}=\delta_{X}^{\mathrm{opt}}$. This stochastic reduced model is constructed with 80 elastic modes of the mean model and is solved by the Monte Carlo method with 1,000 independent realizations and with a frequency resolution of $1 \mathrm{~Hz}$. The confidence region of the modulus of the random FRF (frequency response function) at each observation point defined in Section 4.1 is calculated by using the sample quantiles [39] with a probability level $P_{c}=0.98$. Figure 4 (left and right) and Figure 5 (left and right) display the comparisons between the mean model response predictions, the reference responses of the real system and the confidence region predictions calculated with the stochastic reduced model constructed with the parametric probabilistic approach of modelparameter uncertainties. Figures 4 and 5 show that the confidence region is centered around the resonances of the mean model. This kind of result is general with the parametric probabilistic approach. The confidence region is spanned by the movements of the resonances of the mean model around the nominal values. This is the reason why the parametric probabilistic approach of model-parameter uncertainties does not allow modeling errors to be taken into account. For instance, it can be seen in Figure 4 right that the mean model does not have resonance between $700 \mathrm{~Hz}$ and $1,000 \mathrm{~Hz}$ while the real system as many resonances. It can then be seen that the responses of the real system do not belong to the confidence region spanned by the parametric probabilistic approach in this frequency band $[700,1000] \mathrm{Hz}$ and for the observation point $P_{3}$. The introduction of the nonparametric probabilistic approach of modeling errors coupled with the present probabilistic parametric approach of model-parameter uncertainties will allow the predictions to be improved.

\subsection{Estimation of the dispersion parameters of the prior probability model of model uncertainties}

We consider the stochastic reduced model constructed (1) with the parametric probabilistic approach of uncertain parameter $X$ for which the probabilistic model has been defined 

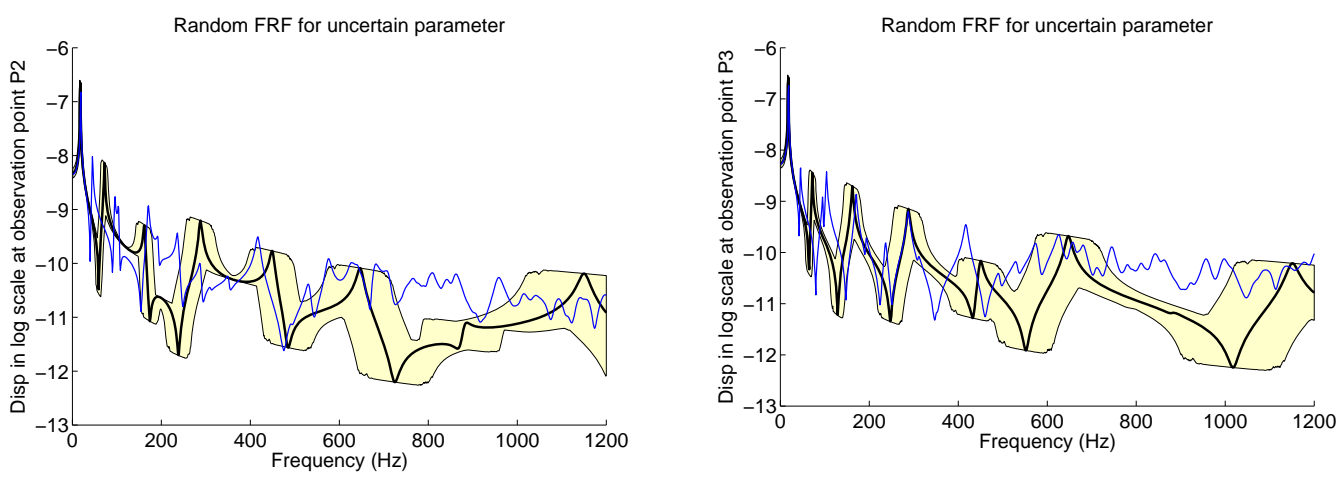

Figure 4. Confidence region calculated with the stochastic reduced model due to uncertain parameter $X$ for $\delta_{X}=0.093$ and probability level $P_{c}=0.98$ (yellow or grey region), reference response (thin solid line), mean model (thick solid line). Observation points $P_{2}$ (left) and $P_{3}$ (right).
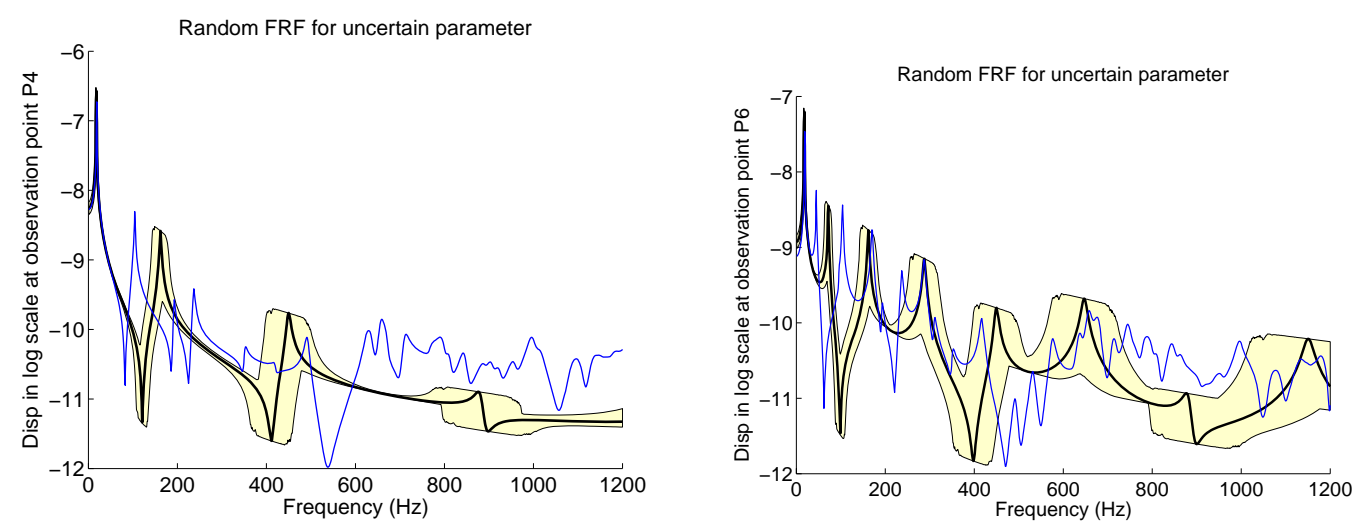

Figure 5. Confidence region calculated with the stochastic reduced model due to uncertain parameter $X$ for $\delta_{X}=0.093$ and probability level $P_{c}=0.98$ (yellow or grey region), reference response (thin solid line), mean model (thick solid line). Observation point $P_{4}$ (left) and $P_{6}$ (right).

in Section 4.4 with $\delta_{X}=\delta_{X}^{\mathrm{opt}}$ and (2) with the nonparametric probabilistic approach of modeling errors for the mass and stiffness operators. Consequently, the dispersion parameters describing the statistical fluctuations induced by modeling errors (see Sections 2.3 .4 and 3) are $\boldsymbol{\delta}_{\mathbf{G}}=\left(\delta_{M}, \delta_{K}\right)$.

The method used for the identification of dispersion parameters $\delta_{M}$ and $\delta_{K}$ is presented in Section 3.2 for the random vector $\mathbf{Z}=\left(Z_{1}, \ldots, Z_{\mu^{\prime}}\right)$ whose components are the random coordinates in the statistical reduction of the random frequency response functions sampled with the frequency resolution of $4 \mathrm{~Hz}$ and for the 6 observation points $P_{1}$ to $P_{6}$. The corresponding value for the reference solution (the real system) is $\mathbf{z}^{\text {ref }}=\left(z_{1}^{\text {ref }}, \ldots, z_{\mu^{\prime}}^{\text {ref }}\right)$. For fixed values of $\delta_{X}, \delta_{M}$ and $\delta_{K}$, the stochastic reduced model is constructed with 80 elastic modes of the mean model and is solved by the Monte Carlo method with 300 independent realizations and with a frequency resolution of $4 \mathrm{~Hz}$. For $\delta_{X}=\delta_{X}^{\mathrm{opt}}$ and for all the trial values 
of $\delta_{M}$ and $\delta_{K}$ belonging to the admissible set, Figure 6 left shows the convergence of the statistical reduction in function of reduced order $\mu^{\prime}$ (see Eq. (45)). This figure shows that a reasonable value for convergence is reached for $\mu^{\prime}=15$. The Neperian logarithm of the likelihood function is defined (see Eq. (46)) by

$$
\mathcal{L}\left(\delta_{M}, \delta_{K}\right)=\sum_{j=1}^{\mu^{\prime}}\left\{\ln \left(p_{Z_{j}}\left(z_{j}^{\mathrm{ref}} ; \delta_{X}^{\mathrm{opt}}, \delta_{M}, \delta_{K}\right)\right)\right\}
$$

in which, for all $j$ in $\left\{1, \ldots \mu^{\prime}\right\}$, the probability density function $z \mapsto p_{Z_{j}}\left(z ; \delta_{X}^{\text {opt }}, \delta_{M}, \delta_{K}\right)$ depends on the known parameter $\delta_{X}^{\text {opt }}$ and on the unknown parameters $\delta_{M}$ and $\delta_{K}$ which have to be identified. This likelihood function is estimated with the stochastic reduced computational model and with the numerical parameters defined above. The optimization problem (see Eq. (47))

$$
\left(\delta_{M}^{\mathrm{opt}}, \delta_{K}^{\mathrm{opt}}\right)=\arg \max _{\left(\delta_{M}, \delta_{K}\right)} \mathcal{L}\left(\delta_{M}, \delta_{K}\right),
$$

is solved by the trial method. Figure 6 right shows for $\delta_{M}$ belonging to the set $\{0.2,0.3,0.4,0.5,0.6,0.7,0.8,0.9,0.97\}$ the graphs of functions $\delta_{K} \mapsto \mathcal{L}\left(\delta_{M}, \delta_{K}\right)$. The optimal values are then $\delta_{M}^{\mathrm{opt}}=0.9$ and $\delta_{K}^{\mathrm{opt}}=0.15$.
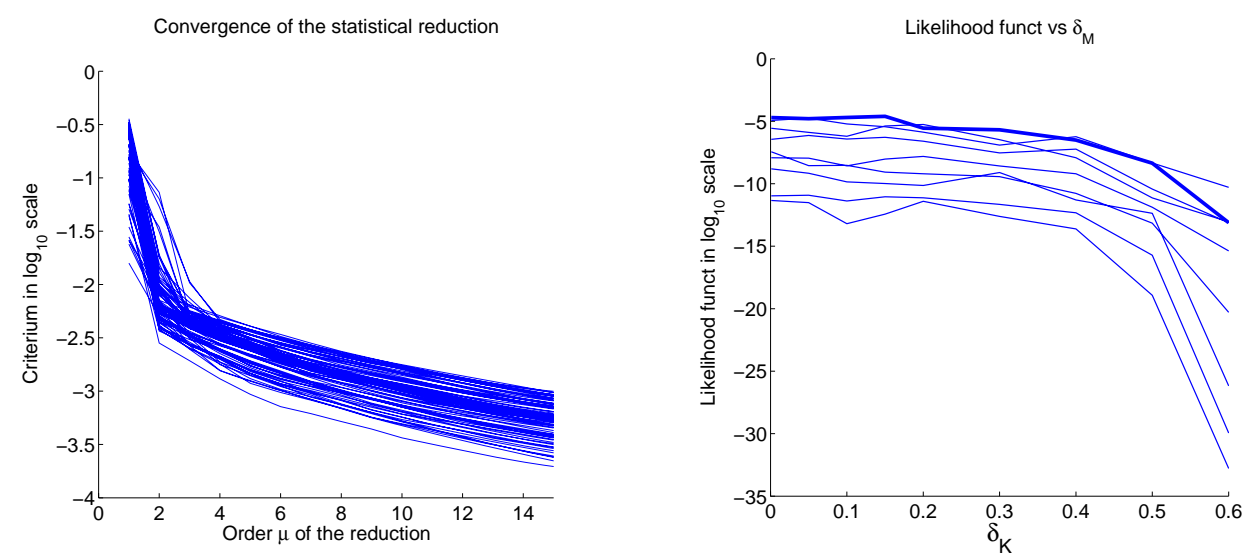

Figure 6. Left Figure: Convergence of the statistical reduction as a function of reduced order $\mu^{\prime}$ and for all the trial values of $\delta_{M}$ and $\delta_{K}$. Right Figure: Graph of $\delta_{K} \mapsto \mathcal{L}\left(\delta_{M}, \delta_{K}\right)$ for $\delta_{M}$ varying from 0.2 to 0.97 (thin solid lines) and for $\delta_{M}=0.90$ (thick solid line).

We now consider the stochastic reduced model constructed with 80 elastic modes of the mean model and for $\delta_{X}=\delta_{X}^{\mathrm{opt}}, \delta_{M}=\delta_{M}^{\mathrm{opt}}$ and $\delta_{K}=\delta_{K}^{\mathrm{opt}}$. The stochastic equation is solved by the Monte Carlo method with 1,000 independent realizations and with a frequency resolution of $1 \mathrm{~Hz}$. The confidence region of the modulus of the random FRF (frequency response function) at each observation point defined in Section 4.1 is calculated as previously by using the sample quantiles with a probability level $P_{c}=0.98$. Figure 7 (left and right) and Figure 8 (left and right) display the comparisons between the mean model response predictions, the reference responses of the real system and the confidence region predictions calculated with 
the stochastic reduced model constructed with the parametric probabilistic approach of modelparameter uncertainties for $\delta_{X}=0.093$ and with the nonparametric probabilistic approach of modeling errors for $\delta_{M}=0.9$ and $\delta_{K}=0.15$. These figures show that the coupling of the two probabilistic approaches for model-parameter uncertainties and modeling errors allow the quality of the prediction to be considerably improved. The method proposed allows the role played by each type of uncertainties to be separately quantified.
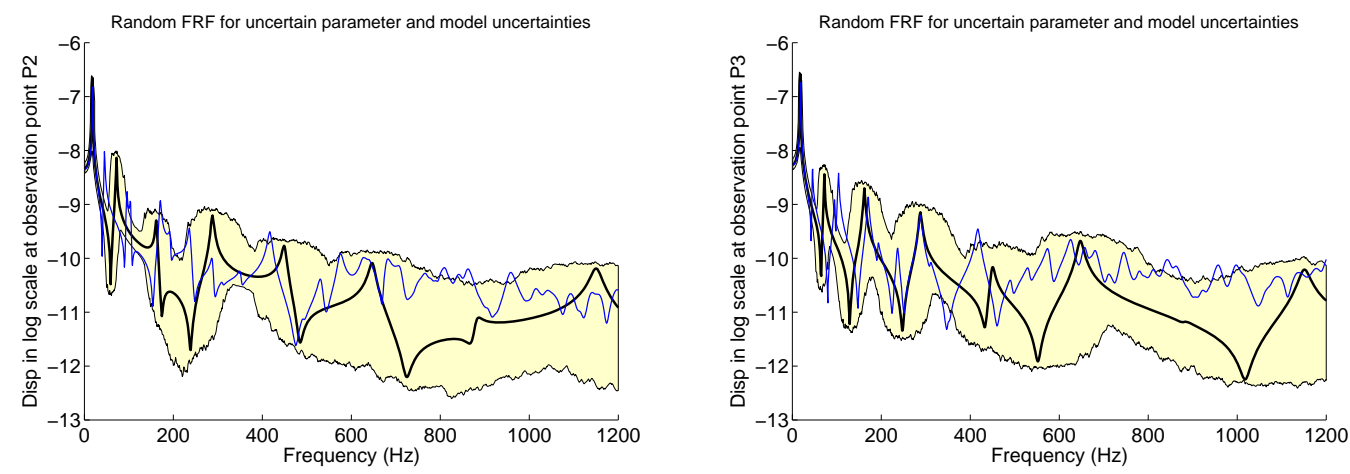

Figure 7. Confidence region with a probability level $P_{c}=0.98$, calculated with the stochastic reduced model including uncertain model parameter for $\delta_{X}=0.093$ and modeling errors for $\delta_{M}=0.9$ and $\delta_{K}=0.15$ (yellow or grey region), reference response (thin solid line), mean model (thick solid line).

Observation points $P_{2}$ (left) and $P_{3}$ (right).


Figure 8. Confidence region with a probability level $P_{c}=0.98$, calculated with the stochastic reduced model including uncertain model parameter for $\delta_{X}=0.093$ and modeling errors for $\delta_{M}=0.9$ and $\delta_{K}=0.15$ (yellow or grey region), reference response (thin solid line), mean model (thick solid line).

Observation points $P_{4}$ (left) and $P_{6}$ (right). 
5. Representation of propagation of uncertainties using polynomial chaos decomposition with random coefficients

In this section the random response of the uncertain dynamical system is represented using the polynomial chaos decomposition with random coefficients [51]. It should be noted that the decomposition proposed is not usual. With the usual decomposition [16], the coefficients are deterministic. Presently, we propose to develop the random response in polynomial chaos only with respect to the random variable $\mathbf{X}$ relative to model-parameter uncertainties. Consequently, if there were no model uncertainties, the coefficients of such a decomposition would be deterministic. Since there are model uncertainties, these coefficients become random vectors which are statistically independent of $\mathbf{X}$. Consequently, the polynomial chaos decomposition with random coefficients which is proposed allows the propagation of uncertainties to be analyzed in separating the propagation of model-parameter uncertainties from the propagation of model uncertainties (modeling errors).

\subsection{Polynomial chaos decomposition with random coefficients}

Let us consider the random variable $\mathbf{X}$ relative to model-parameter uncertainties and introduced in Sections 2.3.1 and 2.3.3. This $\mathbb{R}^{n_{p}}$-valued random variable defined on probability space $(\Theta, \mathcal{T}, \mathcal{P})$ has a probability distribution defined by the probability density function $p_{\mathbf{X}}\left(\mathbf{x} ; \boldsymbol{\delta}_{\mathbf{X}}\right)$ whose support is $\mathcal{C}_{\text {par }}$. It is assumed that there exists a mapping $\xi \mapsto \mathbf{x}=\mathbf{h}(\xi)$ from $\mathbb{R}^{\mu "}$ on $\mathcal{C}_{\text {par }} \subset \mathbb{R}^{n_{p}}$ such that random variable $\mathbf{X}$ can be written as

$$
\mathbf{X}=\mathbf{h}(\boldsymbol{\Xi}) \quad \text { with } \quad \mu " \leq \mu,
$$

in which $\Xi=\left(\Xi_{1}, \ldots, \Xi_{\mu}\right)$ is a $\mathbb{R}^{\mu \prime}$-valued normalized Gaussian random variable defined on $(\Theta, \mathcal{T}, \mathcal{P})$ (the components are independent and each component is a centered Gaussian random variable with a standard deviation equal to one).

Let us assume that $\boldsymbol{\delta}_{\mathbf{X}}$ and $\boldsymbol{\delta}_{\mathbf{G}}$ are given (for instance are equal to the optimal values $\boldsymbol{\delta}_{\mathbf{X}}^{\text {opt }}$ and $\left.\boldsymbol{\delta}_{\mathbf{G}}^{\text {opt }}\right)$. For $\theta$ in $\Theta$ and for $\theta^{\prime}$ in $\Theta^{\prime}$, let $\boldsymbol{\Xi}(\theta)$ be the realization of $\boldsymbol{\Xi}$ and let $\mathbf{X}(\theta)=\mathbf{h}(\boldsymbol{\Xi}(\theta))$ be the corresponding realization of $\mathbf{X}$. Let $Y\left(\theta, \theta^{\prime}\right)$ be the realization of the $\mathbb{R}^{s}$-valued random variable $\boldsymbol{Y}$ defined in Section 3.2 and computed in using Eqs. (39) and (40). From Eqs. (42) and (43), it can be deduced that the realization of the approximation $\Psi^{\mu}$ of $\Psi$ is written as

$$
\mathbb{Y}^{\mu^{\prime}}\left(\theta, \theta^{\prime}\right)=\mathrm{m}_{\mathbb{Y}}+\sum_{j=1}^{\mu^{\prime}} \sqrt{\lambda_{j}} Z_{j}\left(\theta, \theta^{\prime}\right) \varkappa^{j},
$$

in which the components of the realization $\mathbf{Z}\left(\theta, \theta^{\prime}\right)=\left(Z_{1}\left(\theta, \theta^{\prime}\right), \ldots, Z_{\mu^{\prime}}\left(\theta, \theta^{\prime}\right)\right)$ of $\mathbf{Z}$ are such that

$$
Z_{j}\left(\theta, \theta^{\prime}\right)=\frac{1}{\sqrt{\lambda_{j}}}<\mathbb{Y}\left(\theta, \theta^{\prime}\right)-\mathrm{m}_{\mathbb{Y}}, \mathfrak{k}^{j}>.
$$

For $\theta^{\prime}$ fixed in $\Theta^{\prime}$, the polynomial chaos decomposition of the $\mathbb{R}^{\mu^{\prime}}$-valued random variable $\mathbf{Z}\left(., \theta^{\prime}\right)=\left\{\theta \mapsto \mathbf{Z}\left(\theta, \theta^{\prime}\right)\right)$ defined on $(\Theta, \mathcal{T}, \mathcal{P})$ with respect to the $\mathbb{R}^{\mu \prime}$-valued normalized Gaussian random variable $\boldsymbol{\Xi}$ defined on $(\Theta, \mathcal{T}, \mathcal{P})$ is such that

$$
\mathbf{Z}\left(\theta, \theta^{\prime}\right)=\sum_{\boldsymbol{\alpha},|\boldsymbol{\alpha}|=1, \ldots, \mu \mathbf{Z}} \mathbf{A}^{\boldsymbol{\alpha}}\left(\theta^{\prime}\right) \psi^{\boldsymbol{\alpha}}(\boldsymbol{\Xi}(\theta))
$$


in which the vector $\mathbf{A}^{\boldsymbol{\alpha}}\left(\theta^{\prime}\right)$ in $\mathbb{R}^{\mu^{\prime}}$ is given by

$$
\mathbf{A}^{\boldsymbol{\alpha}}\left(\theta^{\prime}\right)=E\left\{\mathbf{Z}\left(., \theta^{\prime}\right) \psi^{\boldsymbol{\alpha}}(\boldsymbol{\Xi})\right\}=\int_{\Theta} \mathbf{Z}\left(\theta, \theta^{\prime}\right) \psi^{\boldsymbol{\alpha}}(\boldsymbol{\Xi}(\theta)) d \mathcal{P}(\theta)
$$

In Eqs. (51) and (52), $\boldsymbol{\alpha}=\left(\alpha_{1}, \ldots, \alpha_{\mu} \prime\right)$ is the multi-index of length $|\alpha|=\alpha_{1}+\ldots+\alpha_{\mu}$ " and $\psi^{\alpha}(\xi)=\psi^{\alpha_{1}}\left(\xi_{1}\right) \times \ldots \times \psi^{\alpha_{\mu}^{\prime \prime}}\left(\xi_{\mu^{\prime \prime}}\right)$ is the normalized Hermite polynomial in which $\xi=\left(\xi_{1}, \ldots, \xi_{\mu}\right)$. Since $\mathbf{Z}$ is a centered random variable, the polynomial chaos decomposition starts with $|\boldsymbol{\alpha}|=1$. Finally, Eq. (51) yields the polynomial chaos decomposition with random coefficients,

$$
\mathbf{Z}=\sum_{\boldsymbol{\alpha},|\boldsymbol{\alpha}|=1, \ldots, \mu \mathbf{Z}} \mathbf{A}^{\boldsymbol{\alpha}} \psi^{\alpha}(\boldsymbol{\Xi})
$$

in which the family of random variables $\left\{\mathbf{A}^{\boldsymbol{\alpha}}, \boldsymbol{\alpha},|\boldsymbol{\alpha}|=1, \ldots, \mu_{\mathbf{Z}}\right\}$ is independent of the family of random variables $\left\{\psi^{\boldsymbol{\alpha}}(\boldsymbol{\Xi}), \boldsymbol{\alpha},|\boldsymbol{\alpha}|=1, \ldots, \mu_{\mathbf{z}}\right\}$. We now construct a reduced-order model to deduce a polynomial decomposition with orthogonal random coefficients.

Let $\mathbf{B}=\left\{\theta^{\prime} \mapsto \mathbf{B}\left(\theta^{\prime}\right)\right\}$ be the random variable defined on $\left(\Theta^{\prime}, \mathcal{T}^{\prime}, \mathcal{P}^{\prime}\right)$ with values in $\mathbb{R}^{N}$ such that $\mathbf{B}\left(\theta^{\prime}\right)=\left\{\mathbf{A}^{\boldsymbol{\alpha}}\left(\theta^{\prime}\right), \boldsymbol{\alpha}:|\boldsymbol{\alpha}|=1, \ldots, \mu_{\mathbf{Z}}\right\}$. The usual statistical reduction of $\mathbf{B}$ is introduced as in Section 3.2 for $\mathbb{Y}$. Let $\mathbf{m}_{\mathbf{B}}$ be the mean value and let $\left[C_{\mathbf{B}}\right]$ be the covariance matrix of the random vector $\mathbf{B}$ such that

$$
\mathbf{m}_{\mathbf{B}}=E\{\mathbf{B}\} \quad, \quad\left[C_{\mathbf{B}}\right]=E\left\{\left(\mathbf{B}-\mathbf{m}_{\mathbf{B}}\right)\left(\mathbf{B}-\mathbf{m}_{\mathbf{B}}\right)^{T}\right\} \quad .
$$

We then introduce the eigenvalue problem

$$
\left[C_{\mathbf{B}}\right] \mathbf{b}^{j}=\lambda_{j}^{\mathbf{B}} \mathbf{b}^{j}
$$

for which the positive eigenvalues are such that $\lambda_{1}^{\mathbf{B}} \geq \ldots \geq \lambda_{N}^{\mathbf{B}}$. The corresponding eigenvectors $\mathbf{b}^{1}, \ldots, \mathbf{b}^{N}$ in $\mathbb{R}^{N}$ are rewritten, for all $j$, as $\mathbf{b}^{j}=\left\{\mathbf{a}^{\bar{\alpha} j}, \boldsymbol{\alpha}:|\boldsymbol{\alpha}|=1, \ldots, \mu_{\mathbf{Z}}\right\}$. Let $\mu_{\mathbf{B}}$ be an integer such that $\mu_{\mathbf{B}} \ll N$. For all fixed multi-index $\boldsymbol{\alpha}$, the following approximation $\mathbf{A}^{\alpha \mu_{\mathbf{B}}}$ of $\mathbf{A}^{\boldsymbol{\alpha}}$ can be deduced,

$$
\mathbf{A}^{\boldsymbol{\alpha} \mu_{\mathbf{B}}}=E\left\{\mathbf{A}^{\boldsymbol{\alpha}}\right\}+\sum_{j=1}^{\mu_{\mathbf{B}}} \sqrt{\lambda_{j}^{\mathbf{B}}} H_{j} \mathbf{a}^{\boldsymbol{\alpha} j},
$$



$$
H_{j}=\frac{1}{\sqrt{\lambda_{j}^{\mathbf{B}}}}<\mathbf{B}-E\{\mathbf{B}\}, \mathbf{b}^{j}>\quad .
$$

Similarly to Eq. (45), the criterium used for analyzing the convergence of the statistical reduction of $\mathbf{B}$ (as a function of the order $\mu_{\mathbf{B}}$ of the reduction) is written as

$$
\operatorname{criter}_{\mathbf{B}}\left(\mu_{\mathbf{B}}\right)=1-\frac{\sum_{j=1}^{\mu_{\mathbf{B}}} \lambda_{j}^{\mathbf{B}}}{\operatorname{tr}\left[C_{\mathbf{B}}\right]} .
$$

The components of $\mathbf{H}$ are second-order random variables which are centered and uncorrelated, that is to say, are such that

$$
E\{\mathbf{H}\}=0 \quad, \quad E\left\{\mathbf{H} \mathbf{H}^{T}\right\}=\left[I_{\mu_{\mathbf{B}}}\right] .
$$


Substituting Eq. (56) into Eq. (53) yields the following decomposition

$$
\mathbf{Z}=\boldsymbol{\Phi}^{0}(\boldsymbol{\Xi})+\sum_{j=1}^{\mu_{\mathbf{B}}} H_{j} \boldsymbol{\Phi}^{j}(\boldsymbol{\Xi})
$$

in which the vector-valued polynomials $\boldsymbol{\Phi}^{j}(\boldsymbol{\Xi})$ of the normalized Gaussian vector $\boldsymbol{\Xi}$ are such that

$$
\begin{gathered}
\boldsymbol{\Phi}^{0}(\boldsymbol{\Xi})=\sum_{\boldsymbol{\alpha},|\boldsymbol{\alpha}|=1, \ldots, \mu_{\mathbf{Z}}} E\left\{\mathbf{A}^{\boldsymbol{\alpha}}\right\} \psi^{\boldsymbol{\alpha}}(\boldsymbol{\Xi}), \\
\boldsymbol{\Phi}^{j}(\boldsymbol{\Xi})=\sqrt{\lambda_{j}^{\mathbf{B}}} \sum_{\boldsymbol{\alpha},|\boldsymbol{\alpha}|=1, \ldots, \mu_{\mathbf{Z}}} \mathbf{a}^{\boldsymbol{\alpha} j} \psi^{\boldsymbol{\alpha}}(\boldsymbol{\Xi}), \quad j=1, \ldots, \mu_{\mathbf{B}} .
\end{gathered}
$$

Note that Eq. (60) corresponds to the polynomial Chaos decomposition with random coefficients representing the propagation of model-parameter uncertainties (polynomial chaos $\boldsymbol{\Phi}^{j}(\boldsymbol{\Xi})$ ) and the propagation of modeling errors (random coefficients $\mathbf{H}_{j}$ which verify Eq. (59)). It should be noted that, for all $\theta$ in $\Theta$ and $\theta^{\prime}$ in $\Theta^{\prime}$, the realization of $\mathbf{Z}$ given by Eq. (60) is such that

$$
\mathbf{Z}\left(\theta, \theta^{\prime}\right)=\boldsymbol{\Phi}^{0}(\boldsymbol{\Xi}(\theta))+\sum_{j=1}^{\mu_{\mathbf{B}}} H_{j}\left(\theta^{\prime}\right) \boldsymbol{\Phi}^{j}(\boldsymbol{\Xi}(\theta))
$$

Finally, substituting Eq. (60) into Eq. (42) or Eq. (49) yields the polynomial Chaos decomposition with random coefficients of the approximation $\mho^{\mu^{\prime}}$ of stochastic response $\mathbb{Y}$ which is written as

$$
\mathbb{Y}^{\mu^{\prime}}=\mathrm{m}_{\mathbb{Y}}+\mathbb{W}^{0}(\boldsymbol{\Xi})+\sum_{j=1}^{\mu_{\mathbf{B}}} H_{j} \mathbb{W}^{j}(\boldsymbol{\Xi})
$$

in which the vector-valued polynomials $\mathbb{W}^{j}(\boldsymbol{\Xi})$ of the normalized Gaussian vector $\boldsymbol{\Xi}$ are such that, for all $j=0,1, \ldots, \mu_{\mathbf{B}}$, we have

$$
\mathbb{W}^{j}(\boldsymbol{\Xi})=\sum_{\ell=1}^{\mu^{\prime}} \sqrt{\lambda_{\ell}} \Phi_{\ell}^{j}(\boldsymbol{\Xi}) \varkappa^{\ell} .
$$

\subsection{Validation}

The configuration, data and results of the example presented in Section 4 are reused below. We then have $n=80$ elastic modes for the reduced mean model. The dispersion parameters are $\delta_{X}=0.093$ for the random variable $X$ (model-parameter uncertainties) and $\delta_{M}=0.9, \delta_{D}=0$ and $\delta_{K}=0.15$ for the dispersion parameter of the random matrices (modeling errors). The observation points are $P_{2}, P_{3}, P_{4}$ and $P_{6}$. The frequency band of analysis is $\left.] 0,1200\right] \mathrm{Hz}$ and the frequency resolution is $4 \mathrm{~Hz}$ (thus, there are 300 frequency sampling points). Consequently, the dimension $s$ of the observation vector $\Upsilon$ is $s=300 \times 4=1200$.

The Monte Carlo numerical simulation is used to solve the stochastic computational model with 20,000 independent realizations in $\left(\theta, \theta^{\prime}\right)$ corresponding to $n_{s}=100$ realizations in $\theta$ and $n_{s}^{\prime}=200$ realizations in $\theta^{\prime}$. The theory presented in Section 5.1 is then applied with $n_{p}=\mu^{\prime \prime}=1$, with $\mu^{\prime}=15$ for the reduction order of $\checkmark$ defined by Eq. (49), with $\mu_{\mathbf{Z}}=8$ for the maximum order of the polynomial chaos decomposition defined by Eq. (51) and with 


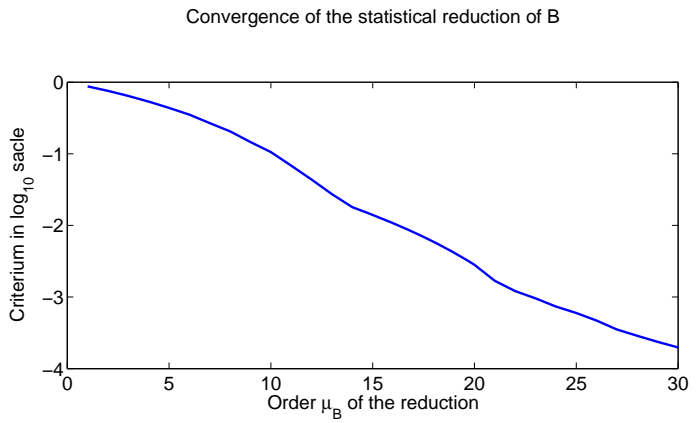

Figure 9. Convergence of the statistical reduction of $\mathbf{B}$ as a function of reduced order $\mu_{\mathbf{B}}$.
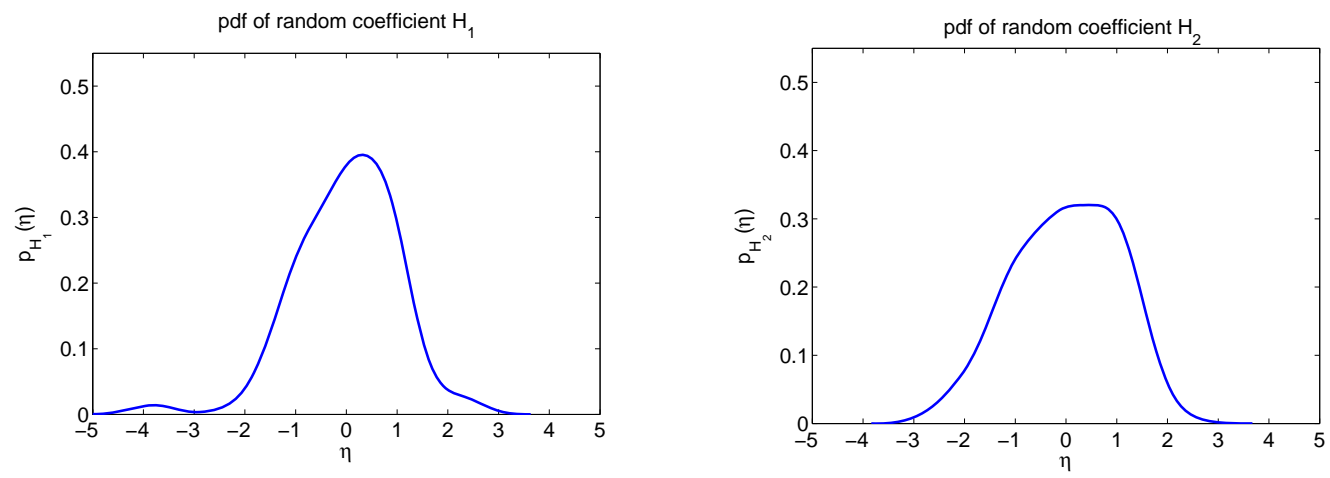

Figure 10. Probability density functions of random coefficients $H_{1}$ (left) and $H_{2}$ (right).
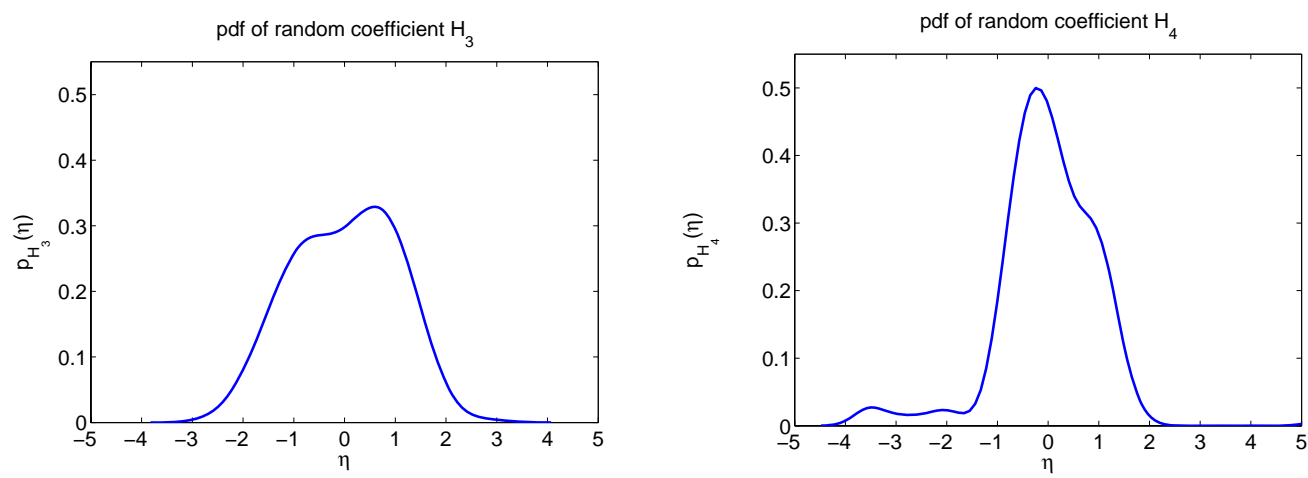

Figure 11. Probability density functions of random coefficients $H_{3}$ (left) and $H_{4}$ (right).

$N=8 \times 15=120$ for the dimension of $\mathbf{B}$. Figure 9 displays the graph of $\mu_{\mathbf{B}} \mapsto \operatorname{criter}_{\mathbf{B}}\left(\mu_{\mathbf{B}}\right)$ defined by Eq. (58) relative to the convergence of the statistical reduction of $\mathbf{B}$ defined by Eq. (56) as a function of reduced order $\mu_{\mathbf{B}}$. Convergence is reasonably reached for $\mu_{\mathbf{B}}=30$. 

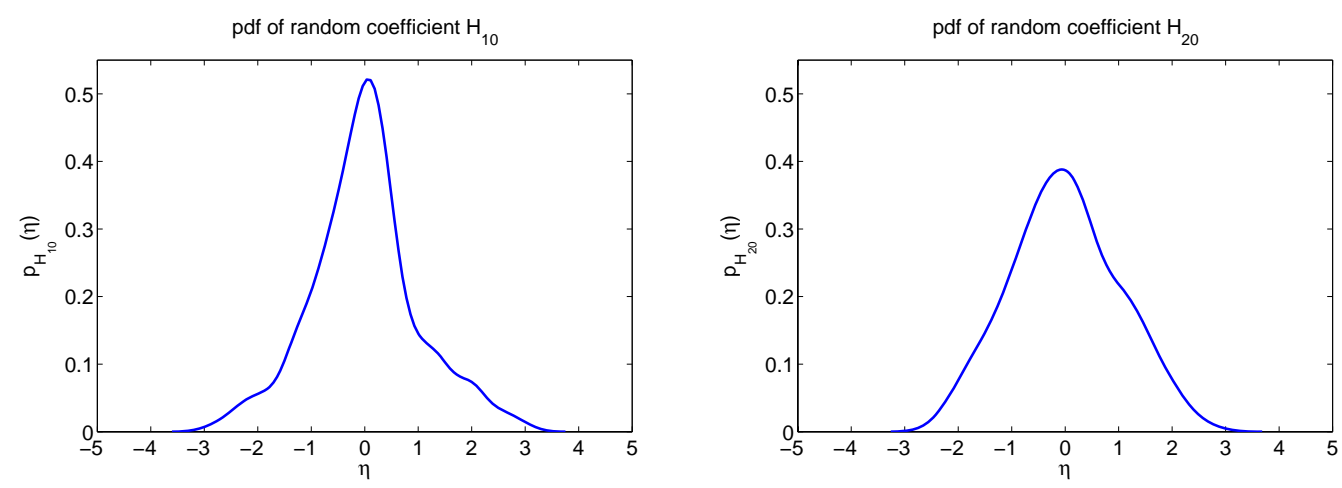

Figure 12. Probability density functions of random coefficients $H_{10}$ (left) and $H_{20}$ (right).

The probability density functions of random variables $H_{1}, \ldots, H_{\mu_{\mathbf{B}}}$ are estimated using the independent realizations of Eq. (57) constructed with the Monte Carlo simulation and a normal kernel function [5] as a smoothing technique. As an illustration, Figures 10, 11 and 12 (left and right) display the graphs of the probability density functions for random variables $H_{1}$, $\mathrm{H}_{2}, \mathrm{H}_{3}, \mathrm{H}_{4}, \mathrm{H}_{10}$ and $\mathrm{H}_{20}$.

6. Future perspective for the construction of a posterior probability model of the polynomial chaos decomposition with random coefficients

Let $\mathbb{Y}^{\mu^{\prime}}$ be the approximation defined by Eq. (49) of the $\mathbb{R}^{s}$-valued random variable $\mathbb{Y}$ defined in Section 3.2. The random vector $\mathbb{Y}^{\mu^{\prime}}$ is expressed in terms of the polynomial chaos decomposition with random coefficients defined by Eq. (60) of the random variable $\mathbf{Z}=\left(Z_{1}, \ldots, Z_{\mu^{\prime}}\right)$ as a function of the normalized Gaussian random vector $\boldsymbol{\Xi}$ and of the random vector $\mathbf{H}=\left(H_{1}, \ldots, H_{\mu_{\mathbf{B}}}\right)$. The probability density function of $\boldsymbol{\Xi}$ with respect to $d \boldsymbol{\xi}$ is $p_{\boldsymbol{\Xi}}(\boldsymbol{\xi})=(2 \pi)^{-\mu " / 2} \exp \left(-\|\boldsymbol{\xi}\|^{2} / 2\right)$. The conditional probability density function $p_{\mathbf{Z} \mid \mathbf{H}}(\mathbf{z} \mid \boldsymbol{\eta})$ of $\mathbf{Z}$ for given $\mathbf{H}=\boldsymbol{\eta}$ and the prior probability density function $p_{\mathbf{H}}^{\text {prior }}(\boldsymbol{\eta})$ of $\mathbf{H}$ can be estimated using Eq. (60) and the prior stochastic computational model presented in Section 2.3.7 as explained in Section 5.1. It should be noted that for the construction of $p_{\mathbf{Z} \mid \mathbf{H}}(\mathbf{z} \mid \eta)$, the stochastic solver can be either the Galerkin method with the polynomial chaos [16] or the Monte Carlo method. In addition, as soon as the prior probability density function $p_{\mathbf{H}}^{\text {prior }}(\boldsymbol{\eta})$ has been estimated, then any probabilistic quantities related to $\Upsilon^{\mu^{\prime}}$ can be estimated using Eqs. (49) and (60) and using a random generator of independent realizations for $\mathbf{H}$ and $\boldsymbol{\Xi}$ as shown by Eq. (63). For instance, the probabilistic quantity of interest is written $\mathbf{J}=E\left\{\widetilde{\mathbf{g}}\left(\mathbb{Y}^{\mu^{\prime}}\right)\right\}$ in which $\widetilde{\mathbf{g}}$ is a given vector-valued function defined on $\mathbb{R}^{s}$. Using Eqs. (49) and (60), this quantity can be rewritten as $\mathbf{J}=E\{\mathbf{g}(\mathbf{H}, \boldsymbol{\Xi})\}$ in which $(\boldsymbol{\eta}, \boldsymbol{\xi}) \mapsto \mathbf{g}(\boldsymbol{\eta}, \boldsymbol{\xi})$ is a given vector-valued function defined on $\mathbb{R}^{\mu_{\mathbf{B}}} \times \mathbb{R}^{\mu}$ ". Consequently, the value of $\mathbf{J}$ calculated with the prior probability model 
of uncertainties and denoted by $\mathbf{J}^{\text {prior }}$ can be written as

$$
\mathbf{J}^{\text {prior }}=\int_{\mathbb{R}^{\mu_{\mathbf{B}}}} \int_{\mathbb{R}^{\mu}} \mathbf{g}(\boldsymbol{\eta}, \boldsymbol{\xi}) p_{\mathbf{H}}^{\text {prior }}(\boldsymbol{\eta}) p_{\Xi}(\boldsymbol{\xi}) d \boldsymbol{\eta} d \boldsymbol{\xi} .
$$

Let us assumed that one experimental data Yobserved of the $\mathbb{R}^{s}$-valued random variable $\mathbb{Y}$ defined in Section 3.2 is available. Taking into account Eq. (50), the corresponding experimental value of $\mathbf{Z}$ is denoted by $\mathbf{Z}^{\text {observed }}=\left(Z_{1}^{\text {observed }}, \ldots, Z_{\mu^{\prime}}^{\text {observed }}\right)$ and can be estimated by

$$
Z_{j}^{\text {observed }}=\frac{1}{\sqrt{\lambda_{j}}}<\mathbb{Y}^{\text {observed }}-\mathrm{m}_{\mathbb{Y}}, \mathrm{x}^{j}>\quad .
$$

The objective is to propose an approach based on the use of the Bayes method (see for instance $[22],[52])$ to construct a posterior probability density function $p_{\mathbf{H}}^{\text {post }}(\boldsymbol{\eta})$ of $\mathbf{H}$ using the information defined by $\mathbf{Z}^{\text {observed }}$ in order to improve the value $\mathbf{J}^{\text {prior }}$ denoted $\mathbf{J}^{\text {post }}$ and such that

$$
\mathbf{J}^{\text {post }}=\int_{\mathbb{R}^{\mu_{\mathbf{B}}}} \int_{\mathbb{R}^{\mu^{\prime \prime}}} \mathbf{g}(\boldsymbol{\eta}, \boldsymbol{\xi}) p_{\mathbf{H}}^{\text {post }}(\boldsymbol{\eta}) p_{\Xi}(\boldsymbol{\xi}) d \boldsymbol{\eta} d \boldsymbol{\xi}
$$

Using the Bayes formula yields

$$
p_{\mathbf{H}}^{\text {post }}(\boldsymbol{\eta})=c_{0} p_{\mathbf{H}}^{\text {prior }}(\boldsymbol{\eta}) p_{\mathbf{Z} \mid \mathbf{H}}\left(\mathbf{Z}^{\text {observed }} \mid \boldsymbol{\eta}\right)
$$

in which $c_{0}$ is a constant of normalization. Substituting Eq. (67) into Eq. (66)yields

$$
\mathbf{J}^{\text {post }}=c_{0} \int_{\mathbb{R}^{\mu_{\mathbf{B}}}} \int_{\mathbb{R}^{\mu \prime}} \mathbf{g}(\boldsymbol{\eta}, \boldsymbol{\xi}) p_{\mathbf{H}}^{\text {prior }}(\boldsymbol{\eta}) p_{\mathbf{Z} \mid \mathbf{H}}\left(\mathbf{Z}^{\text {observed }} \mid \boldsymbol{\eta}\right) p_{\Xi}(\boldsymbol{\xi}) d \boldsymbol{\eta} d \xi
$$

Denoting by $E_{\Xi}$ the mathematical expectation corresponding to the integration with respect to the random variable $\boldsymbol{\Xi}$ for which the probability distribution is $p_{\boldsymbol{\Xi}}(\boldsymbol{\xi}) d \boldsymbol{\xi}$ and $E_{\mathbf{H}}^{\text {prior }}$ the mathematical expectation corresponding to the integration with respect to the random variable $\mathbf{H}$ for which the probability distribution is $p_{\mathbf{H}}^{\text {prior }}(\boldsymbol{\eta}) d \boldsymbol{\eta}$, Eq. (68) can be rewritten as

$$
\mathbf{J}^{\text {post }}=c_{0} E_{\Xi} E_{\mathbf{H}}^{\text {prior }}\left\{\mathbf{g}(\mathbf{H}, \boldsymbol{\Xi}) p_{\mathbf{Z} \mid \mathbf{H}}\left(\mathbf{Z}^{\text {observed }} \mid \mathbf{H}\right)\right\} .
$$

Taking $\mathbf{g}(\mathbf{H}, \boldsymbol{\Xi})=1$ in Eq. (69) yields $1=c_{0} E_{\mathbf{H}}^{\text {prior }}\left\{p_{\mathbf{Z} \mid \mathbf{H}}\left(\mathbf{Z}^{\text {observed }} \mid \mathbf{H}\right)\right\}$ and consequently, Eq. (69) can be rewritten as

$$
\mathbf{J}^{\text {post }}=\frac{E_{\Xi} E_{\mathbf{H}}^{\text {prior }}\left\{\mathbf{g}(\mathbf{H}, \boldsymbol{\Xi}) p_{\mathbf{Z} \mid \mathbf{H}}\left(\mathbf{Z}^{\text {observed }} \mid \mathbf{H}\right)\right\}}{E_{\mathbf{H}}^{\text {prior }}\left\{p_{\mathbf{Z} \mid \mathbf{H}}\left(\mathbf{Z}^{\text {observed }} \mid \mathbf{H}\right)\right\}} .
$$

Concerning the calculation of Eq. (70), the Monte Carlo numerical simulation can be used. Let $\boldsymbol{\Xi}\left(\theta_{1}\right), \ldots, \boldsymbol{\Xi}\left(\theta_{n_{s}}\right)$ be $n_{s}$ independent realizations of the random variable $\boldsymbol{\Xi}$ following the probability distribution $p_{\Xi}(\boldsymbol{\xi}) d \xi$. Let $\mathbf{H}\left(\theta_{1}^{\prime}\right), \ldots, \mathbf{H}\left(\theta_{n_{s}}^{\prime}\right)$ be $n_{s}^{\prime}$ independent realizations of the random variable $\mathbf{H}$ following the probability distribution $p_{\mathbf{H}}^{\text {prior }}(\boldsymbol{\eta}) d \boldsymbol{\eta}$. For $n_{s}$ and $n_{s}^{\prime}$ sufficiently large, an approximation of Eq. (70) is given by

$$
\mathbf{J}^{\text {post }}=\simeq \frac{\frac{1}{n_{s} n_{s}^{\prime}} \sum_{j=1}^{n_{s}} \sum_{j^{\prime}=1}^{n^{\prime}} \mathbf{g}\left(\mathbf{H}\left(\theta_{j^{\prime}}^{\prime}\right), \boldsymbol{\Xi}\left(\theta_{j}\right)\right) p_{\mathbf{Z} \mid \mathbf{H}}\left(\mathbf{Z}^{\text {observed }} \mid \mathbf{H}\left(\theta_{j^{\prime}}^{\prime}\right)\right.}{\frac{1}{n_{s}^{\prime}} \sum_{j^{\prime}=1}^{n_{s}^{\prime}} p_{\mathbf{Z} \mid \mathbf{H}}\left(\mathbf{Z}^{\text {observed }} \mid \mathbf{H}\left(\theta_{j^{\prime}}^{\prime}\right)\right.} .
$$

The estimation of $p_{\mathbf{Z} \mid \mathbf{H}}\left(\mathbf{Z}^{\text {observed }} \mid \mathbf{H}\left(\theta_{j^{\prime}}^{\prime}\right)\right.$ is performed using again Monte Carlo simulation with a double loops on $j$ an $j^{\prime}$ and using Eq. (63). 


\section{Conclusions}

We have presented a new generalized probabilistic approach to take into account modelparameter uncertainties and modeling errors in computational model for structural linear dynamics. This approach can easily be extended to computational linear vibroacoustics and to computational nonlinear structural dynamics. This method allows a prior probability model of model-parameter uncertainties and a prior probability model of modeling errors to be separately constructed. When a few experimental data are available, a procedure for the identification of the dispersion parameters of the prior probability models of uncertainties is proposed. A complete validation has been given and clearly show the advantage of the method proposed with respect to the usual output-prediction-error method. A chaos decomposition with random coefficients has also been proposed to represent the prior probabilistic model of random responses in separating the propagation of model-parameter uncertainties and the propagation of modeling errors in the computational model. Such a construction gives future perspectives to improve the prior probability model of uncertainties in constructing a posterior probability model as soon as experimental data are available.

\section{REFERENCES}

[1]. Anderson, T.W. (1958). Introduction to Multivariate Statistical Analysis (John Wiley \& Sons, New York).

[2]. Babuska, I., Tempone, R. and Zouraris, G.E. (2005). Solving elliptic boundary value problems with uncertain coefficients by the finite element method: the stochastic formulation, Computer Methods in Applied Mechanics and Engineering 194(12-16), 1251-1294.

[3]. Babuska, I., Nobile, F. and Tempone R. (2007). A stochastic collocation method for elliptic partial differential equations with random input data, SIAM Journal on Numerical Analysis 45(3), $1005-1034$.

[4]. Beck, J.L. and Katafygiotis, L.S. (1998). Updating models and their incertainties. I: Bayesian statistical framework, Journal of Engineering Mechanics 124(4), 455-461.

[5]. Bowman, A. W. and Azzalini, A. (1997). Applied Smoothing Techniques for Data Analysis, Oxford University Press.

[6]. Capiez-Lernout, E. and Soize, C. (2008). Robust design optimization in computational mechanics, Journal of Applied Mechanics - Transactions of the ASME 75(2), 0210011-02100111.

[7]. Chebli, H. and Soize, C. (2004). Experimental validation of a nonparametric probabilistic model of non homogeneous uncertainties for dynamical systems, J. Acoust. Soc. Am. 115(2) 697-705.

[8]. Chen, C., Duhamel, D. and Soize, C. (2006). Probabilistic approach for model and data uncertainties and its experimental identification in structural dynamics: Case of composite sandwich panels, Journal of Sound and Vibration 294(1-2), 64-81.

[9]. Das, S., Ghanem, R. and Spall, J.C. (2007). Asymptotic sampling distribution for polynomial chaos representation from data: a maximum entropy and fisher information approach, SIAM Journal on Scientific Computing 30(5), 2207-2234.

[10]. Desceliers, C., Ghanem, R. and Soize, C. (2006). Maximum likelihood estimation of stochastic chaos representations from experimental data, International Journal for Numerical Methods in Engineering 66(6), 978-1001.

[11]. Desceliers, C., Soize, C. and Cambier, S. (2004) Non-parametric - parametric model for random uncertainties in nonlinear structural dynamics - Application to earthquake engineering, Earthquake Engineering and Structural Dynamics 33(3), 315-327.

[12]. Duchereau, J. and Soize, C. (2006). Transient dynamics in structures with nonhomogeneous uncertainties induced by complex joints, Mechanical Systems and Signal Processing 20(4), 854-867.

[13]. Durand, J.-F., Soize, C. and Gagliardini, L. (2008). Structural-acoustic modeling of automotive vehicles in presence of uncertainties and experimental identification and validation, J. Acoust. Soc. Am. 124(3), 1513-1525.

[14]. Fernandez, C., Soize, C. and Gagliardini, L. (2009). Fuzzy structure theory modeling of sound-insulation layers in complex vibroacoustic uncertain sytems - Theory and experimental validation, J. Acoust. Soc. Am. 125(1), 138-153.

[15]. Fougeaud, C. and Fuchs, A. (1967). Statistique (Dunod, Paris) (2nd edition 1972). 
[16]. Ghanem, R. and Spanos, P.D (2003). Stochastic Finite Elements: A Spectral Approach, revised edition (Dover Publications, New-York) (originally published in 1991, Springer-Verlag, New York).

[17]. Ghanem, R. and Red-Horse J. (1999). Propagation of probabilistic uncertainty in complex physical systems using a stochastic finite element approach, Physica D 133(1-4), 137-144.

[18]. Ghanem, R., Masri, S., Pellissetti, M. et al (2005). Identification and prediction of stochastic dynamical systems in a polynomial chaos basis, Computer Methods in Applied Mechanics and Engineering 194(12-16), 1641-1654.

[19]. Ghanem, R. and Ghosh, D. (2007). Efficient characterization of the random eigenvalue problem in a polynomial chaos decomposition, International Journal for Numerical Methods in Engineering 72(4), 486504 (2007).

[20]. Ghosh, D. and Farhat, C. (2008). Strain and stress computation in stochastic finite element methods, International Journal for Numerical Methods in Engineering 74(8), 1219-1239.

[21]. Jaynes, E.T. (1957). Information theory and statistical mechanics, Physical Review 106(4), 620-630 and $\mathbf{1 0 8}(2), 171-190$.

[22]. Kaipo J. and Somersalo E. (2005). Statistical and Computational Inverse Problems (Springer, New York).

[23]. Kapur, J.N. and Kesavan H.K. (1992). Entropy Optimization Principles with Applications (Academic Press, San Diego).

[24]. Lagaros, N.D. and Papadrakakis, M. (2007). Robust seismic design optimization of steel structures, Structural and Multidisciplinary Optimization 33(6), 457-469.

[25]. Langley, R. (2000). Unified approach to probabilistic and possibilistic analysis of uncertain systems, Journal of Engineering Mechanics 126(11), 1163-1172.

[26]. Le Maitre, O.P., Knio, O.M., Najm, H.N. et al. (2004). Uncertainty propagation using Wiener-Haar expansions, Journal of Computational Physics 197(1), 28-57.

[27]. Mace, R.R., Worden, W., Manson, G. (editors) (2005). Uncertainty in structural dynamics, Special issue of the Journal of Sound and Vibration 288(3), 431-790.

[28]. MacKeown, P.K. (1997). Stochastic Simulation in Physics (Springer-Verlag, Singapore).

[29]. Mehta, M.L. (1991). Random Matrices, Revised and Enlarged Second Edition (Academic Press, San Diego).

[30]. Mignolet, M.P. and Soize, C. (2008). Nonparametric stochastic modeling of linear systems with prescribed variance of several natural frequencies, Probabilistic Engineering Mechanics, 23(2-3), 267-278.

[31]. Mignolet, M.P. and Soize, C. (2008). Stochastic reduced order models for uncertain nonlinear dynamical systems, Computer Methods in Applied Mechanics and Engineering, 197(45-48), 3951-3963.

[32]. Nouy, A., Clement, A., Schoefs, F. et al (2008). An extented stochastic finite element method for solving stochastic partial differential equations on random domains, Computer Methods in Applied Mechanics and Engineering 197(51-52), 4663-4682.

[33]. Pradlwarter, H.J., Schueller, G.I. and Szekely, G.S. (2002). Random eigenvalue problems for large systems, Computer and Structures, 80, 2415-2424.

[34]. Rubinstein R.Y. (1981), Simulation and the Monte Carlo Method, John Wiley and Sons, New York.

[35]. Sampaio, R., Soize, C. (2007). On measures of non-linearity effects for uncertain dynamical systems Application to a vibro-impact system, Journal of Sound and Vibration 303(3-5), 659-674.

[36]. Schueller, G.I. (2005) eds. Computational methods in stochastic mechanics and reliability analysis, Computer Methods in Applied Mechanics and Engineering 194(12-16), 1251-1795.

[37]. Schueller, G.I. (2007). On the treatment of uncertainties in structural mechanics and analysis, Computer and Structures 85(5-6), 235-243.

[38]. Schueller, G.I. and Jensen, H.A. (2008). Computational methods in optimization considering uncertainties - An overview, Computer Methods in Applied Mechanics and Engineering 198(1), 2-13.

[39]. Serfling, R.J. (1980). Approximation Theorems of Mathematical Statistics (John Wiley \& Sons, New York).

[40]. Shannon, C.E. (1948). A mathematical theory of communication, Bell System Technology Journal $\mathbf{2 7}$ $379-423$ and $623-659$.

[41]. Soize, C. (2000). A nonparametric model of random uncertainties for reduced matrix models in structural dynamics, Probabilistic Engineering Mechanics 15(3), 277-294.

[42]. Soize, C. (2001). Maximum entropy approach for modeling random uncertainties in transient elastodynamics, Journal of the Acoustical Society of America 109(5), 1979-1996.

[43]. Soize, C. (2003). Random matrix theory and non-parametric model of random uncertainties in vibration analysis, Journal of Sound and Vibration 263, 893-916.

[44]. Soize C, Ghanem R. (2004). Physical systems with random uncertainties : Chaos representation with arbitrary probability measure, SIAM Journal On Scientific Computing 26(2), 395-410.

[45]. Soize, C. (2005). A comprehensive overview of a non-parametric probabilistic approach of model uncertainties for predictive models in structural dynamics, Journal of Sound and Vibration 233(3), 623652 . 
[46]. Soize, C. (2005). Random matrix theory for modeling random uncertainties in computational mechanics, Computer Methods in Applied Mechanics and Engineering 194(12-16), 1333-1366.

[47]. Soize, C. (2006). Non Gaussian positive-definite matrix-valued random fields for elliptic stochastic partial differential operators, Computer Methods in Applied Mechanics and Engineering 195, 26-64.

[48]. Soize, C. (2008). Construction of probability distributions in high dimension using the maximum entropy principle. Applications to stochastic processes, random fields and random matrices, International Journal for Numerical Methods in Engineering 76(10), 1583-1611.

[49]. Soize, C., Capiez-Lernout, E. and Ohayon, R. (2008). Robust updating of uncertain computational models using experimental modal analysis, AIAA Journal 46(11), 2955-2965.

[50]. Soize, C., Capiez-Lernout, E., Durand, J.-F., Fernandez, C. and Gagliardini, L. (2008). Probabilistic model identification of uncertainties in computational models for dynamical systems and experimental validation, Computer Methods in Applied Mechanics and Engineering 198(1), 150-163.

[51]. Soize, C. and Ghanem, R. (2009). Reduced chaos decomposition with random coefficients of vector-valued random variables and random fields, Computer Methods in Applied Mechanics and Engineering, 198(2126), 1926-1934.

[52]. Spall, J.C. (2003). Introduction to Stochastic Search and Optimization (John Wiley \& Sons, Hoboken).

[53]. Walter, E. and Pronzato, L. (1997). Identification of Parametric Models from Experimental Data (Springer-Verlag, Berlin). 